\title{
Nonlinear interactions in deformable container cranes
}

\author{
Andrea Arena, Walter Lacarbonara ${ }^{\dagger}$ Matthew P. Cartmell ${ }^{\ddagger}$
}

July 27,2016

\begin{abstract}
Nonlinear dynamic interactions in harbour quayside cranes due to a two-to-one internal resonance between the lowest bending mode of the deformable boom and the in-plane pendular mode of the container are investigated. To this end, a three-dimensional model of container cranes accounting for the elastic interaction between the crane boom and the container dynamics is proposed. The container is modelled as a three-dimensional (3D) rigid body elastically suspended through hoisting cables from the trolley moving along the crane boom modelled as an Euler-Bernoulli beam. The reduced governing equations of motion are obtained through the Euler-Lagrange equations employing the boom kinetic and stored energies, derived via a Galerkin discretisation based on the mode shapes of the two-span crane boom used as trial functions, and the kinetic and stored energies of the rigid body container and the elastic hoisting cables. First, conditions for the onset of internal resonances between the boom and the container are found. A higher order perturbation treatment of the Taylor expanded equations of motion in the neighbourhood of a two-to-one internal resonance between the lowest boom bending mode and the lowest pendular mode of the container is carried out. Continuation of the fixed points of the modulation equations together with stability analysis yields a rich bifurcation behaviour, which features Hopf bifurcations. It is shown that consideration of higher

*Research Associate, Department of Structural and Geotechnical Engineering, Sapienza University, Rome, 00184, Italy, email: andrea.arena@uniroma1.it

$\dagger$ Associate Professor, Department of Structural and Geotechnical Engineering, Sapienza University, Rome, 00184, Italy, email: walter.lacarbonara@uniroma1.it

‡Professor, Department of Mechanical Engineering, University of Sheffield, Sheffield S1 3JD, England, UK, email:
\end{abstract} m.cartmell@sheffield.ac.uk 
order terms (cubic nonlinearities) beyond the quadratic geometric and inertia nonlinearities breaks the symmetry of the bifurcation equations, shifts the bifurcation points and the stability ranges, and leads to bifurcations not predicted by the low order analysis.

Keywords: Crane dynamics; Perturbation analysis; Modal autoparametric interactions; Twoto-one internal resonances; Higher order approximation; Boom elasticity.

\section{Introduction}

A rich variety of factors can influence the dynamics of container crane systems, such as the trolley motion during working maneuvers or wind-induced excitations [2, 3]. A typical modelling approach for describing the container crane dynamics relies on the assumption of undeformability of the crane supporting structure, of which the boom is the main elastic component. However, this assumption is not always valid since the dynamics of the boom undergoing bending motion in the vertical plane may interact with the dynamics of the suspended container in specific configurations. Indeed, the trolley vertical motion induced by the boom elastodynamic response acts as a parametric excitation for the payload. In particular, resonant autoparametric couplings may be induced if the vertical bending oscillation frequency of the two-span boom is in a suitable ratio with the frequency of one of the container/payload modes. Different modelling approaches are proposed in the literature such as those based on simplified one-degree-of-freedom (one-dof) pendulum models. Although largely employed, these models are not appropriate to describe the overall dynamics of a container crane multibody system, and to capture the geometric nonlinearities affecting the container motion and arising from the hoisting system. A refined planar model was proposed in [13] where a double pendulum, two-dimensional (2D) model was adopted although neglecting the hoisting cables deformability. Planar (2D) pendulum-type crane models were also employed to study payload oscillations control via a nonclassical vibration absorber in [12], via an active open-loop strategy [20] or fuzzy controls using a variable truss geometry [8].

A three-dimensional (3D) analytical model of suspended containers was first proposed in [9] where the motion of the container was described accounting for the deformability of the hoisting cables and for disturbances. A comprehensive classification of these models is given in [1] where applications and limitations of the different theories are discussed. A recent work [3] provided a detailed and accurate fully nonlinear analytical model of the dynamics of container cranes subject to external wind excitations and arbitrary maneuvers and discussing the effects 
of the hoisting cables elasticity. Among other features, in [3] the theoretical studies were corroborated by experimental results. In consonance with what experimentally and numerically demonstrated in [3], the dynamic behaviour of a container crane system is characterised by lightly damped motions which can show rich frequency content, characteristic of a typical nonlinear behaviour, when large-amplitude oscillations are induced through some resonance mechanism.

The majority of the works available in the literature, in particular those based on analytical modelling, typically neglect the dynamics of the crane structure (i.e., the supporting frame and boom) which, instead, should be taken into account when the flexibility of the boom can affect the payload oscillations or when seismic excitations cannot be neglected. A theoretical study of the kinematics of a typical multi-cable suspension system was proposed in [15] and in [7] the same authors illustrated the steps necessary to model the dynamics of a rubbertyred gantry crane and discussed various modelling criteria with emphasis on the importance of nonlinear coupling between the chosen coordinates. In $[5,6]$, modelling of a variety of pendular structures was proposed and suitable control strategies were thoroughly examined by highlighting the computational implications of the models on the control implementations. In [21] the dynamic behaviour of gantry cranes was investigated taking into account also the flexibility of the crane structure, and in [4] a six-dof analytical model was developed to investigate the behaviour of container cranes under seismic excitation. In [19] a finite element model of a quayside crane was employed to show that, for particular crane configurations, the analysis of container oscillations should take into account the boom deformability.

In the present work, a multi-dof model describing the dynamics of container crane systems is presented. The likelihood of dynamic interactions between the deformable boom and the container is investigated considering all possible operational positions of the container and payload masses. In particular, 2:1 and 3:1 ratios between the fundamental boom frequency and the in-plane and out-of-plane container pendular frequencies are found as potential triggers of internal resonances for particular container crane configurations. For two-to-one internal resonances, the method of multiple scales $[17,18,16]$ is employed to obtain second and third order asymptotic expansions of the crane response. A thorough bifurcation analysis carried out via path-following of the fixed points of the modulation equations sheds light onto several interesting features of the crane coupled-mode responses. 


\section{Mechanical formulation of container cranes with deformable boom}

The proposed mechanical model of container cranes is based on a fully nonlinear kinematic formulation to describe the overall 3D motion and accounts for the elasticity of the hoisting cables. A key novel aspect, with respect to the formulation recently proposed by the same authors in $[2,3]$, is the deformability of the crane boom that can interact with the suspended container motion. The deformability of the cantilever boom can play an important role in the dynamics of the suspended container since particular configurations of the container crane system may lead to nonlinear interactions (i.e., transfers of energy) between the boom dynamics and the container pendular motion.

Figure 1 (top) shows a typical crane employed in harbour quays to move containers in and out of cargo ships while Fig. 1 (bottom) portrays a schematic representation in the vertical plane where most of the container motion occurs. The boom geometric and mechanical characteristics are such that the truss girder presents negligible deformations in the horizontal plane, whereas its deformability in the vertical plane gives rise to low-frequency, flexural oscillations.

When the frequency of the boom bending oscillations is in a suitable ratio with one of the container natural frequencies, internal resonance phenomena can take place. In particular, when the ratio between the boom lowest frequency and the container pendular frequency is 2:1, the directly excited boom motion can suffer saturation so that, upon increasing the excitation amplitude, energy is transferred into large-amplitude pendular oscillations of the container.

Next, the derivation of the nonlinear equations of motion of the container crane system accounting for the crane boom deformability is shown. The truss girder of the crane is modelled as an Euler-Bernoulli beam whose equivalent stiffness and inertial characteristics are extracted from the geometry and the elasticity of a quayside-crane (Fantuzzi-Reggiane crane manufacturer) utilised at the Cagliari International Container Terminal (CICT) in Cagliari (Sardinia, Italy). These characteristics have been identified by employing an in-house developed finite element model of the truss girder. The boom deforms in the vertical plane in which it sustains its weight and the moving trolley with the suspended payload. Due to its geometric features, shear deformations can be neglected and thus the Euler-Bernoulli purely flexural behaviour dominates its response. Moreover, due to its stiffness characteristics, the boom does not undergo large-amplitude vibrations which can so be described by the linear Euler-Bernoulli beam 


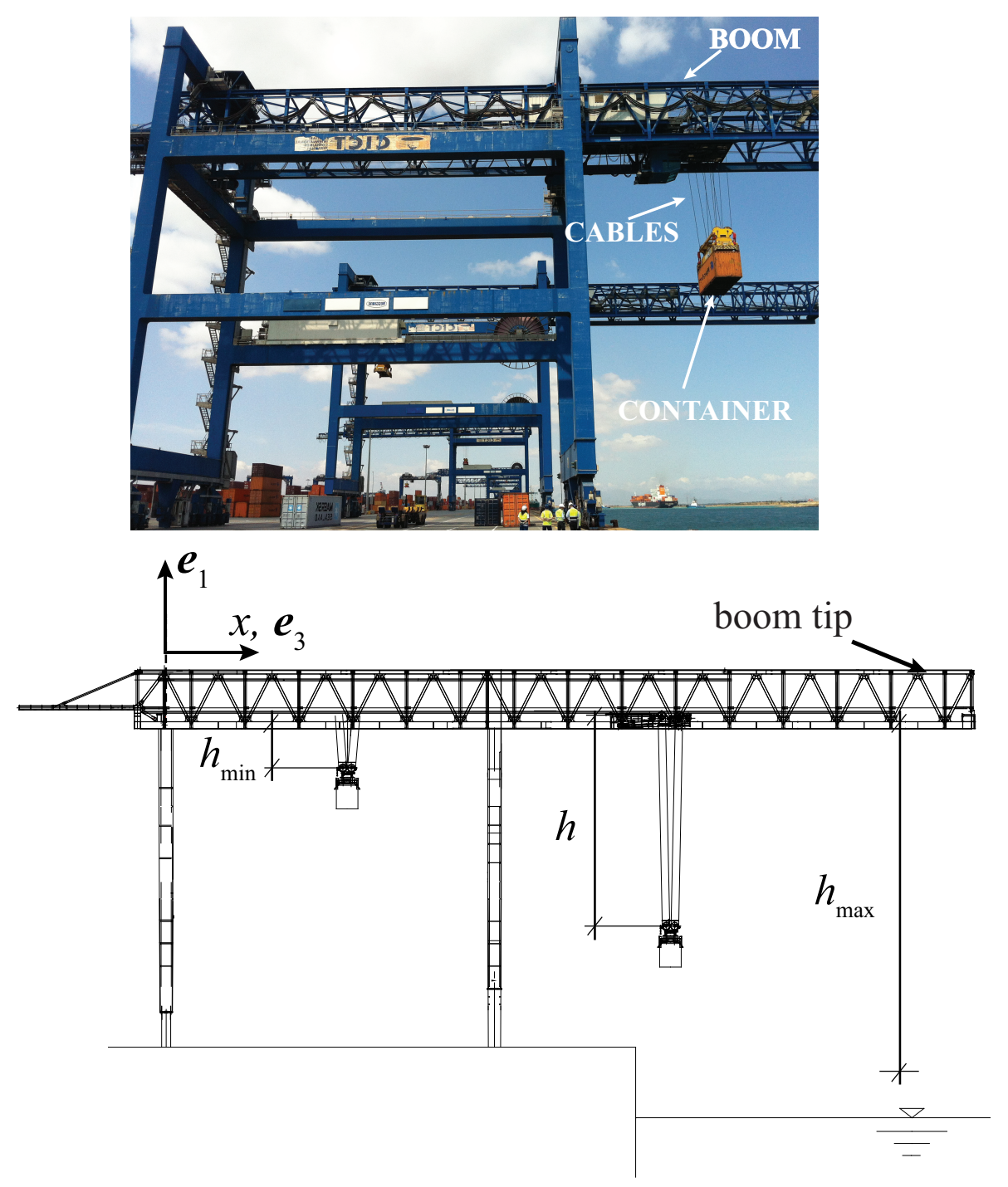

Figure 1: (Top) Quayside crane at CICT in Cagliari (Italy), (bottom) 2D representation of the container crane system.

theory.

On the other hand, the container is modelled as a rigid body elastically suspended from the trolley that can move along rails mounted on the crane boom. The description of the kinematic parameters governing the motion of the container crane system is based on the formulation proposed in [3] enriched by the introduction of the boom elasticity.

\subsection{Three-dimensional kinematic modeling}

Before discussing the kinematics and mechanics of the crane model, few remarks on the adopted notation are provided. Vectors, which are elements of Euclidean 3 -space $\mathbb{E}^{3}$, and vector-valued 
functions are denoted by lower-case, italic, bold-face symbols. The dot product and cross product of (vectors) $\boldsymbol{u}$ and $\boldsymbol{v}$ are denoted $\boldsymbol{u} \cdot \boldsymbol{v}$ and $\boldsymbol{u} \times \boldsymbol{v}$. The length of vector $\boldsymbol{u}$ is denoted by $\|\boldsymbol{u}\|$.

In the fixed Cartesian frame $\left(\boldsymbol{e}_{1}, \boldsymbol{e}_{2}, \boldsymbol{e}_{3}\right)$, with origin in the left terminal section of the crane boom, $\boldsymbol{e}_{1}$ is taken co-linear with the vertical direction and $\boldsymbol{e}_{3}$ is collinear with the crane boom direction in its reference configuration (see Fig. 1). The current crane configuration at time $t$ is described by vectors $\boldsymbol{p}_{b}(x, t)$ and $\boldsymbol{p}_{c}(t)$ denoting, respectively, the position of the trolley moving along the boom centerline and the position of the container centre of mass. Their representation in the frame $\left(\boldsymbol{e}_{1}, \boldsymbol{e}_{2}, \boldsymbol{e}_{3}\right)$ is given as

$$
\begin{aligned}
& \boldsymbol{p}_{b}(x, t)=v(x, t) \boldsymbol{e}_{1}+x \boldsymbol{e}_{3}, \\
& \boldsymbol{p}_{c}(t)=p_{1}(t) \boldsymbol{e}_{1}+p_{2}(t) \boldsymbol{e}_{2}+p_{3}(t) \boldsymbol{e}_{3},
\end{aligned}
$$

where $x$ is the trolley coordinate along the boom centerline, $v(x, t)$ is the vertical displacement of the boom and $p_{i}(t)(i=1,2,3)$ are the components of the container position vector. In this modelling approach, the boom out-of plane dynamics are neglected for the reasons discussed above.

(a)

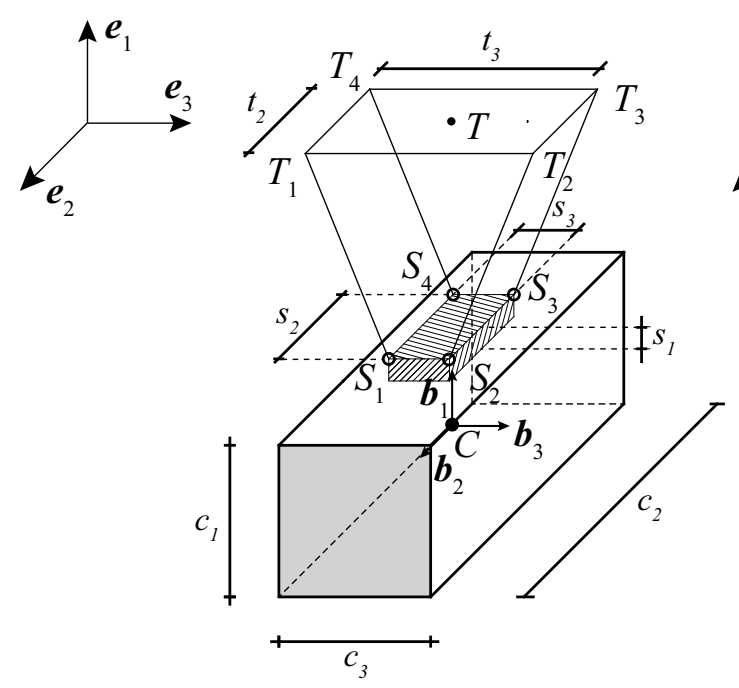

(b)

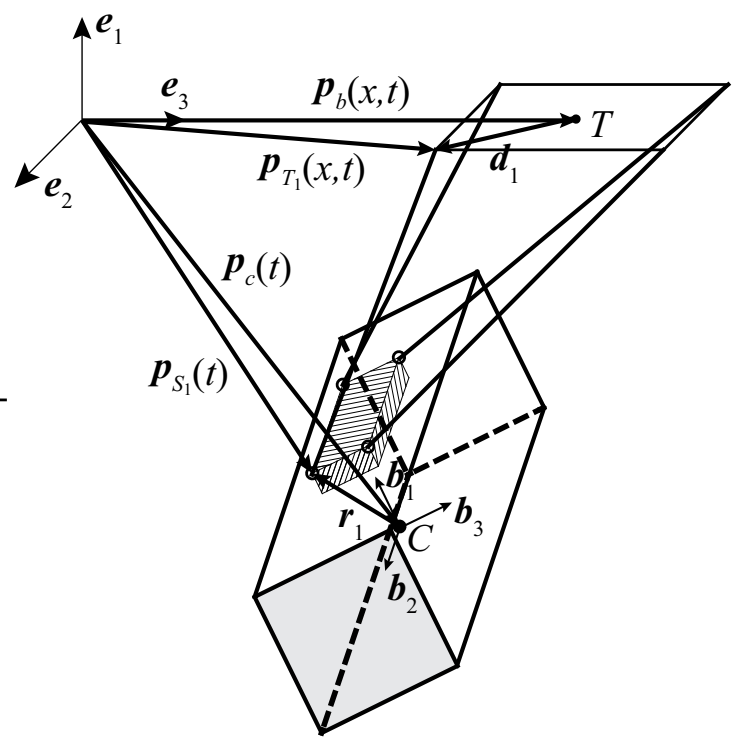

Figure 2: (a) Container reference configuration and (b) current configuration.

The local frame $\left(\boldsymbol{b}_{1}, \boldsymbol{b}_{2}, \boldsymbol{b}_{3}\right)$, having its origin in the container centre of mass $C$, is considered to describe the position vector $\boldsymbol{r}_{i}$ of points $S_{i}(i=1,2,3,4)$ representing the pulleys on the 
spreader bar on the top of the container and its expression is $\boldsymbol{r}_{i}(t)=r_{i, 1} \boldsymbol{b}_{1}+r_{i, 2} \boldsymbol{b}_{2}+r_{i, 3} \boldsymbol{b}_{3}$. Thus, in the current container configuration, the position of $S_{i}$ is given by the vector $\boldsymbol{p}_{S_{i}}(t)=$ $\boldsymbol{p}_{c}(t)+\boldsymbol{r}_{i}(t)$. Similarly, the position of points $T_{i}(i=1,2,3,4)$, denoting the pulleys on the trolley, with respect to the trolley mid-point $T$, are described by $\boldsymbol{d}_{i}=d_{i, 1} \boldsymbol{e}_{1}+d_{i, 2} \boldsymbol{e}_{2}+d_{i, 3} \boldsymbol{e}_{3}$ when the trolley mid-point $T$ lies in the left terminal section of the crane boom; therefore, their position in the current configuration of the trolley can be expressed as $\boldsymbol{p}_{T_{i}}(x, t)=\boldsymbol{p}_{b}(x, t)+\boldsymbol{d}_{i}$. The expressions of the components $r_{i, j}$ and $d_{i, j}(i=1,2,3,4$ and $j=1,2,3)$ depend on the geometric parameters of the container crane and are reported in Tab. 1.

\begin{tabular}{l|cccc}
\hline$i$ & 1 & 2 & 3 & 4 \\
\hline$d_{i, 1}$ & 0 & 0 & 0 & 0 \\
$d_{i, 2}$ & $\frac{t_{2}}{2}$ & $\frac{t_{2}}{2}$ & $-\frac{t_{2}}{2}$ & $-\frac{t_{2}}{2}$ \\
$d_{i, 3}$ & $-\frac{t_{3}}{2}$ & $\frac{t_{3}}{2}$ & $\frac{t_{3}}{2}$ & $-\frac{t_{3}}{2}$ \\
\hline$r_{i, 1}$ & $\frac{c_{1}}{2}+s_{1}$ & $\frac{c_{1}}{2}+s_{1}$ & $\frac{c_{1}}{2}+s_{1}$ & $\frac{c_{1}}{2}+s_{1}$ \\
$r_{i, 2}$ & $\frac{s_{2}}{2}$ & $\frac{s_{2}}{2}$ & $-\frac{s_{2}}{2}$ & $-\frac{s_{2}}{2}$ \\
$r_{i, 3}$ & $-\frac{s_{3}}{2}$ & $\frac{s_{3}}{2}$ & $\frac{s_{3}}{2}$ & $-\frac{s_{3}}{2}$ \\
\hline
\end{tabular}

Table 1: Components of the pulleys position vectors.

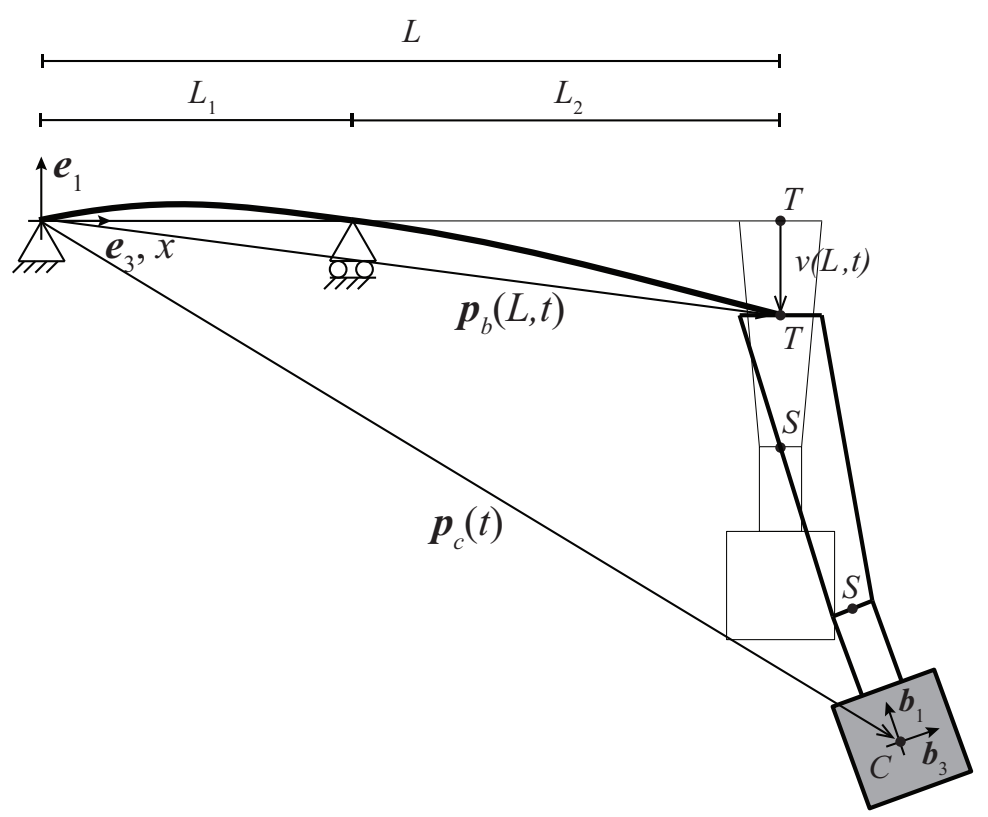

Figure 3: Planar view of the reference (thin lines) and current (thick lines) configurations of the deformable container crane system. 
The rotational motion undergone by the container is described by the orthogonal tensor $\mathbf{R}(t)$, whose expression can be found in $[2,3]$, representing the change of orientation of the frame $\left(\boldsymbol{b}_{1}, \boldsymbol{b}_{2}, \boldsymbol{b}_{3}\right)$ with respect to the fixed frame $\left(\boldsymbol{e}_{1}, \boldsymbol{e}_{2}, \boldsymbol{e}_{3}\right)$. The finite rotations of the frame are parametrized by a sequence of rotations described by the angles $\phi_{1}(t), \phi_{2}(t)$, and $\phi_{3}(t)$. Thus, the container angular velocity vector $\boldsymbol{\omega}_{c}(t)=\omega_{1}(t) \boldsymbol{b}_{1}+\omega_{2}(t) \boldsymbol{b}_{2}+\omega_{3}(t) \boldsymbol{b}_{3}$ can be expressed in terms of nonlinear combination of angular rates $\dot{\phi}_{j}(t)(j=1,2,3)$ as

$$
\begin{aligned}
\boldsymbol{\omega}_{c}(t) & =\left(\dot{\phi}_{1}(t)+\dot{\phi}_{2}(t) \sin \phi_{3}(t)\right) \boldsymbol{b}_{1} \\
& +\left(\dot{\phi}_{2}(t) \cos \phi_{1}(t) \cos \phi_{3}(t)+\dot{\phi}_{3}(t) \sin \phi_{1}(t)\right) \boldsymbol{b}_{2} \\
& +\left(\dot{\phi}_{3}(t) \cos \phi_{1}(t)-\dot{\phi}_{2}(t) \sin \phi_{1}(t) \cos \phi_{3}(t)\right) \boldsymbol{b}_{3},
\end{aligned}
$$

where the overdot indicates differentiation with respect to time $t$ and $\boldsymbol{\omega}_{c}(t)$ satisfies the relationship $\dot{\boldsymbol{b}}_{j}=\boldsymbol{\omega}_{c}(t) \times \boldsymbol{b}_{j}(j=1,2,3)$.

Natural coordinates. By introducing a natural parametrization of the motion of the pendulumlike system here considered, a suitable description of the container crane kinematics can be achieved using the natural coordinates $\left\{l(t), \psi_{2}(t), \psi_{3}(t), \phi_{1}(t), \phi_{2}(t), \phi_{3}(t)\right\}$ whose meaning is shown in Fig. 4.

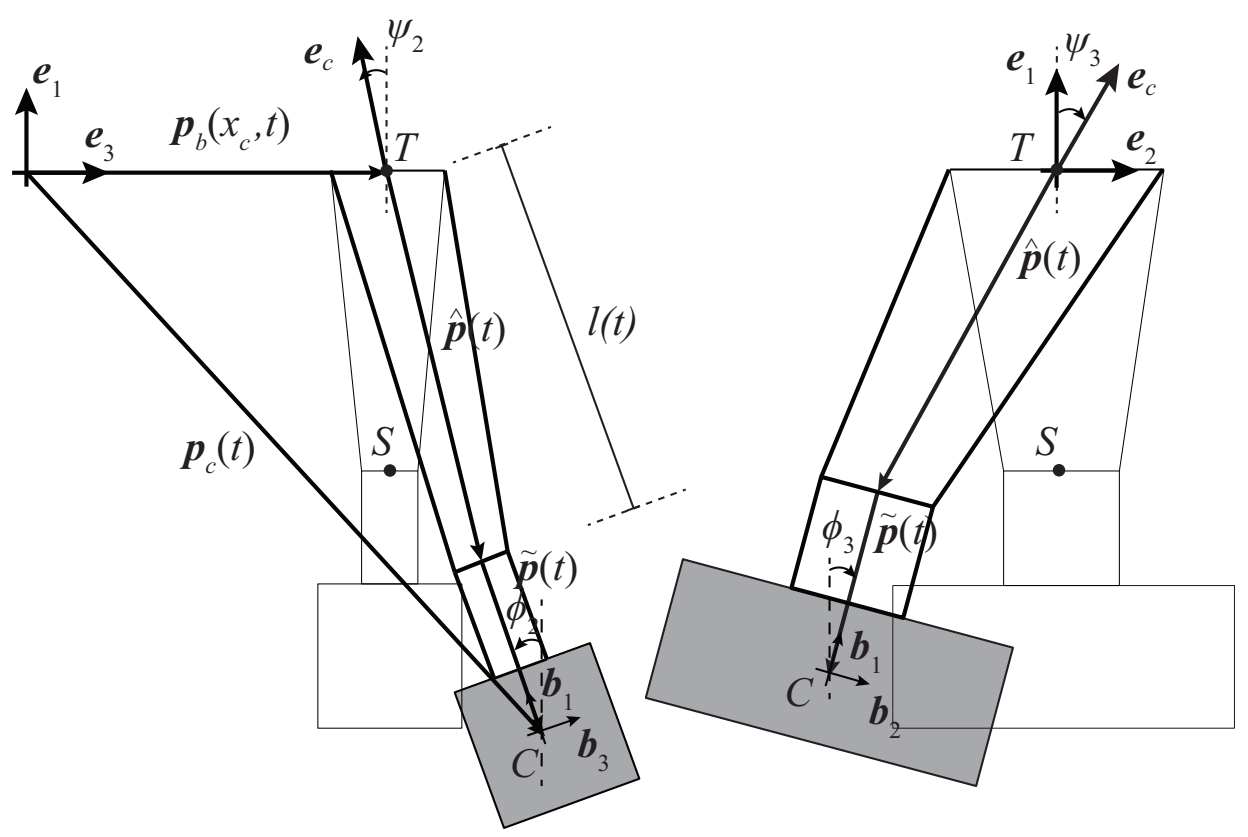

Figure 4: Parameterisation of the crane kinematics based on the choice of natural coordinates.

The unit vector $\boldsymbol{e}_{c}$ can be expressed in terms of the fixed unit vectors as $\boldsymbol{e}_{c}=\cos \psi_{2}(t) \cos \psi_{3}(t) \boldsymbol{e}_{1}+$ 
$\sin \psi_{3}(t) \boldsymbol{e}_{2}-\sin \psi_{2}(t) \cos \psi_{3}(t) \boldsymbol{e}_{3}$. In turn, the vectors $\hat{\boldsymbol{p}}(t)$ and $\tilde{\boldsymbol{p}}(t)$, defined as $\hat{\boldsymbol{p}}(t)=-l(t) \boldsymbol{e}_{c}$ and $\tilde{\boldsymbol{p}}(t)=-\left(\frac{c_{1}}{2}+s_{1}\right) \boldsymbol{b}_{1}$, can be written in the form

$$
\begin{aligned}
& \hat{\boldsymbol{p}}(t)=l(t)\left(-\cos \psi_{2}(t) \cos \psi_{3}(t) \boldsymbol{e}_{1}-\sin \psi_{3}(t) \boldsymbol{e}_{2}+\sin \psi_{2}(t) \cos \psi_{3}(t) \boldsymbol{e}_{3}\right), \\
& \tilde{\boldsymbol{p}}(t)=\left(\frac{c_{1}}{2}+s_{1}\right)\left(-\cos \phi_{2}(t) \cos \phi_{3}(t) \boldsymbol{e}_{1}-\sin \phi_{3}(t) \boldsymbol{e}_{2}+\sin \phi_{2}(t) \cos \phi_{3}(t) \boldsymbol{e}_{3}\right) .
\end{aligned}
$$

Thus, for each fixed position $x_{c}$ of the trolley along the boom, the position vector of the container centre of mass can expressed in terms of natural coordinates as

$$
\boldsymbol{p}_{c}(t)=\boldsymbol{p}_{b}\left(x_{c}, t\right)+\hat{\boldsymbol{p}}(t)+\tilde{\boldsymbol{p}}(t)
$$

\subsection{Equations of motion}

Based on the kinematic model described in the previous section, the equations governing the $3 \mathrm{D}$ motion of the container crane system are obtained using the Euler-Lagrange equations based on the system Lagrangian $\mathcal{L}$ computed below [14]. The stored and kinetic energies associated with the boom motion are computed according to

$$
\begin{aligned}
& V_{b}=\frac{1}{2} \int_{0}^{L} v^{\prime \prime}(x, t) E J v^{\prime \prime}(x, t) d x, \\
& K_{b}=\frac{1}{2} \int_{0}^{L} \dot{v}(x, t) \rho A \dot{v}(x, t) d x,
\end{aligned}
$$

where the prime indicates differentiation with respect to the coordinate $x$ along the boom centerline, $E J$ is the equivalent bending stiffness of the truss girder, $\rho A$ indicates the boom mass per unit reference length, and $L$ is the total boom length. $V_{b}$ is the boom elastic energy stored in pure bending and $K_{b}$ is the purely translational boom kinetic energy in consonance with the Euler-Bernoulli beam theory according to which the deformation is solely characterized by the elastic curvature $\mu=v^{\prime \prime}$ and rotary inertia is neglected.

On the other hand, the container potential and kinetic energies are expressed as

$$
\begin{aligned}
& V_{c}=M g \boldsymbol{p}_{c}(t) \cdot \boldsymbol{e}_{1}, \\
& K_{c}=\frac{1}{2} M \dot{\boldsymbol{p}}_{c}(t) \cdot \dot{\boldsymbol{p}}_{c}(t)+\frac{1}{2} \boldsymbol{\omega}_{c}(t) \cdot\left(\boldsymbol{J}^{\mathrm{M}} \cdot \boldsymbol{\omega}_{c}(t)\right),
\end{aligned}
$$

where $g$ is the gravity acceleration, $M$ is the container mass, and $\boldsymbol{J}^{\mathrm{M}}$ is the tensor of second mass moments whose nontrivial diagonal components $J_{j}^{\mathrm{M}}(j=1,2,3)$ are the second mass moments calculated with respect to the local frame axes $\boldsymbol{b}_{j}$.

By assuming a linearly elastic constitutive law for the hoisting cables, the stored energy is given by

$$
W_{c}=\frac{1}{2} \sum_{i=1}^{4} N_{i}(t) l_{i}(t)\left(\nu_{i}(t)-1\right)
$$


where $l_{i}, N_{i}$, and $\nu_{i}$ are the length, the tension, and the stretch of the $i$ th hoisting cable at time $t$. The tension is constitutively expressed as $N_{i}(t)=E_{c} A_{c}\left(\nu_{i}(t)-1\right)$ where $E_{c}$ is the cables Young's modulus, $A_{c}$ the cables cross-section area, and the nonlinear expression of the stretch is given by

$$
\nu_{i}(t)=\frac{\left\|\boldsymbol{p}_{T_{i}}(t)-\boldsymbol{p}_{S_{i}}(t)\right\|}{l_{i}(t)}, \quad i=1, \ldots, 4
$$

The length $l_{i}(t)$ of the $i$ th cable can be evaluated by prescribing the vertical position $h(t)$ of the container with respect to the boom (i.e., the vertical distance between points $T$ and $S$ ) according to the expression $l_{i}(t)=\left[h(t)^{2}+\left(\frac{t_{2}}{2}-\frac{s_{2}}{2}\right)^{2}+\left(\frac{t_{3}}{2}-\frac{s_{3}}{2}\right)^{2}\right]^{1 / 2}$. Finally, the Lagrangian of the system can be written as $\mathcal{L}=K_{b}+K_{c}-\left(V_{b}+V_{c}+W_{c}\right)$.

Linear viscous damping forces $f_{p_{j}}^{d}(t)$ and couples $c_{\phi_{j}}^{d}(t)$ are taken into account using the expressions:

$$
f_{p_{j}}^{d}(t)=2 \zeta_{c} \omega_{p_{j}} M \dot{p}_{j}(t), \quad c_{\phi_{j}}^{d}(t)=2 \zeta_{c} \omega_{\phi_{j}} J_{j}^{\mathrm{M}} \dot{\phi}_{j}(t), \quad j=1,2,3,
$$

where $\zeta_{c}$ is an equivalent container damping ratio and $\omega_{p_{j}}$ and $\omega_{\phi_{j}}$ are the natural circular frequencies of the $j$ th mode associated to the translational and rotational components of the container motion, respectively. Similarly, the linear damping force associated with the dissipation in the boom is expressed as

$$
f_{b}(t)=2 \zeta_{b} \omega_{b} \frac{1}{2} \int_{0}^{L} \rho A \frac{\partial}{\partial \dot{v}}\left(\dot{v}(x, t)^{2}\right) d x
$$

where $\zeta_{b}$ is the boom damping ratio (in the lowest mode) and $\omega_{b}$ is the natural circular frequency of the lowest vibrational mode of the boom.

Discretisation of the equations of motion. The mechanical modeling of the container crane system features the coupling between a distributed-parameter system, the crane boom, and a six-dof rigid body represented by the container. Therefore, the kinematics of the overall system are described by the container position vector (i.e., the three components $p_{j}(t)$, $j=1,2,3)$, the three container finite rotations $\phi_{j}(t)(j=1,2,3)$ and the boom vertical displacement $v(x, t)$, a function of space and time. In this work, the well-known discretisation technique based on the Faedo-Galerkin method is employed to reduce the space-dependence in the continuum part of the system and, therefore, to reduce the mixed partial-ordinary differential equations of motion of the coupled continuum-discrete system into a set of ordinary differential equations. 
The lowest $N_{m}$ mode shapes of an unshearable beam subject to the boundary conditions of the crane boom (see the schematic representation in Fig. 3) are assumed as trial functions. The boundary conditions for the boom are

$$
\begin{aligned}
& v(0, t)=0=v^{\prime \prime}(0, t), v^{\prime \prime}(L, t)=0=v^{\prime \prime \prime}(L, t), \\
& v\left(L_{1}, t\right)=0, \Delta v\left(L_{1}, t\right)=0, \Delta v^{\prime}\left(L_{1}, t\right)=0, \Delta v^{\prime \prime}\left(L_{1}, t\right)=0,
\end{aligned}
$$

where $\Delta$, applied to the unknown function $v\left(L_{1}, t\right)$ and its derivatives, indicates the jump of the function at $x=L_{1}$ (i.e., the difference between the values attained at $L_{1}^{+}=L_{1}+\varepsilon$ and at $L_{1}^{-}=L_{1}-\varepsilon$ where $\varepsilon$ is an infinitesimal quantity compared to $L_{1}$ ). According to the adopted discretisation procedure, the vertical displacement of the boom can be then expressed as

$$
v(x, t)=\sum_{m=1}^{N_{m}} \bar{v}_{m}(t) \Phi_{m}(x)
$$

where $\Phi_{m}(x)$ represents the $m$ th mode shape of the boom and $\bar{v}_{m}(t)$ is the $m$ th generalized coordinate. By introducing the vector of generalised coordinates

$$
\boldsymbol{\xi}(t)=\left\{\bar{v}_{1}(t), \ldots, \bar{v}_{m}(t), l(t), \psi_{2}(t), \psi_{3}(t), \phi_{1}(t), \phi_{2}(t), \phi_{3}(t)\right\}
$$

it is possible to obtain the equations of motion of the container crane system in the form

$$
\frac{d}{d t}\left(\mathcal{L}_{\dot{\xi}_{k}}\right)-\mathcal{L}_{\xi_{k}}+d_{k} \dot{\xi}_{k}=Q_{k}
$$

where $\xi_{k}$ represents the $k$ th generalized coordinate $\left(k=1, \ldots, 6+N_{m}\right)$, the subscripts in $\mathcal{L}$ denote partial differentiation with respect to the argument indicated by the subscript and $\frac{d}{d t}$ indicates the total derivative with respect to time. The term $d_{k} \dot{\xi}_{k}$ is the $k$ th component of the linear viscous-type generalized force expressed in terms of natural coordinates and derived from Eq. (9). The representation of Eq. (9) in terms of natural coordinates can be obtained by substituting Eq. (4) into Eq. (9) and by linearising the resulting expressions. The right-end side of Eq. (13) is the $k$ th component of the generalized force due to external excitations and its expression will be presented in the following sections.

\subsection{A quayside container crane example: modal characteristics}

The analytical model presented in the previous section is here specialised by taking into account the characteristic parameters of the container cranes employed at the Cagliari International Container Terminal in Cagliari (Sardinia, Italy). The selected quayside crane manufactured 
by the Italian company Fantuzzi-Reggiane is shown in Fig. 1. A $40 \mathrm{ft}$ high cube container, having a maximum mass $M$ of 30 tonnes, is considered in the calculations. The geometric and mechanical characteristics of the case-study container crane are reported in Table 2.

\begin{tabular}{ccccc}
\hline$t_{2}[\mathrm{~m}]$ & $t_{3}[\mathrm{~m}]$ & $s_{1}[\mathrm{~m}]$ & $s_{2}[\mathrm{~m}]$ & $s_{3}[\mathrm{~m}]$ \\
4.896 & 1.93 & 1.95 & 4.8 & 0.95 \\
\hline$c_{1}[\mathrm{~m}]$ & $c_{2}[\mathrm{~m}]$ & $c_{3}[\mathrm{~m}]$ & $\mathrm{N}^{\circ}$ cables $[-]$ & $\zeta_{b}[\%]$ \\
2.896 & 12.192 & 2.4384 & 4 & 0.1 \\
\hline$A_{c}\left[\mathrm{~m}^{2}\right]$ & $E_{c}\left[\mathrm{~N} / \mathrm{m}^{2}\right]$ & $M[\mathrm{~kg}]$ & $J_{1}^{\mathrm{M}}, J_{3}^{\mathrm{M}}\left[\mathrm{kg} \mathrm{\textrm {m } ^ { 2 }}\right]$ & $J_{2}^{\mathrm{M}}\left[\mathrm{kg} \mathrm{m}^{2}\right]$ \\
$7.561 \times 10^{-4}$ & $9 \times 10^{10}$ & 17.7 to $42.7 \times 10^{3}$ & 230 to $560 \times 10^{3}$ & 21 to $51 \times 10^{3}$ \\
\hline$L[\mathrm{~m}]$ & $L_{1}[\mathrm{~m}]$ & $L_{2}[\mathrm{~m}]$ & $\rho A[\mathrm{~kg} / \mathrm{m}]$ & $E J\left[\mathrm{~N} \mathrm{\textrm {m } ^ { 2 } ]}\right.$ \\
90 & 36 & 54 & 2910.17 & $2.5 \times 10^{11}$ \\
\hline
\end{tabular}

Table 2: Characteristic parameters of the Fantuzzi-Reggiane quayside container crane at CICT.

An experimental campaign was carried out on the quayside container crane in [3]. The experiments enabled a parameter identification of the container crane system and a testing of the fidelity of the mechanical model employed in [3] which neglected the deformability of the boom. Together with the main modal features (mass and stiffness) characterising the dynamics of the crane, the crane damping, one of the most uncertain parameters in these lightly damped systems, was evaluated for several container configurations. The experimental results led to the evaluation of the following expression of the container damping factor as a function of the height $h$ :

$$
\begin{aligned}
\zeta_{c}= & 0.0167-2.69 \cdot 10^{-5} h+3.419 \cdot 10^{-7} h^{2} \\
& +4.310 \cdot 10^{-8} h^{3}-4.394 \cdot 10^{-10} h^{4}-9.878 \cdot 10^{-11} h^{5}
\end{aligned}
$$

Moreover, to characterise the overall nonlinear dynamic behaviour of the container crane system, including the onset of internal resonances, the investigation of its modal properties is carried out next. The eigenproblem obtained from Eq. (13) is solved in terms of generalised coordinates collected in $\boldsymbol{\xi}(t)$ to give the $6+N_{m}$ eigenvalues and eigenvectors. These modal features are computed for all possible configurations of the crane. In particular, the effects of the hoisting cables length (here, for convenience, the vertical component $h$ is considered) and the size of the container mass together with its position along the crane boom are investigated. 

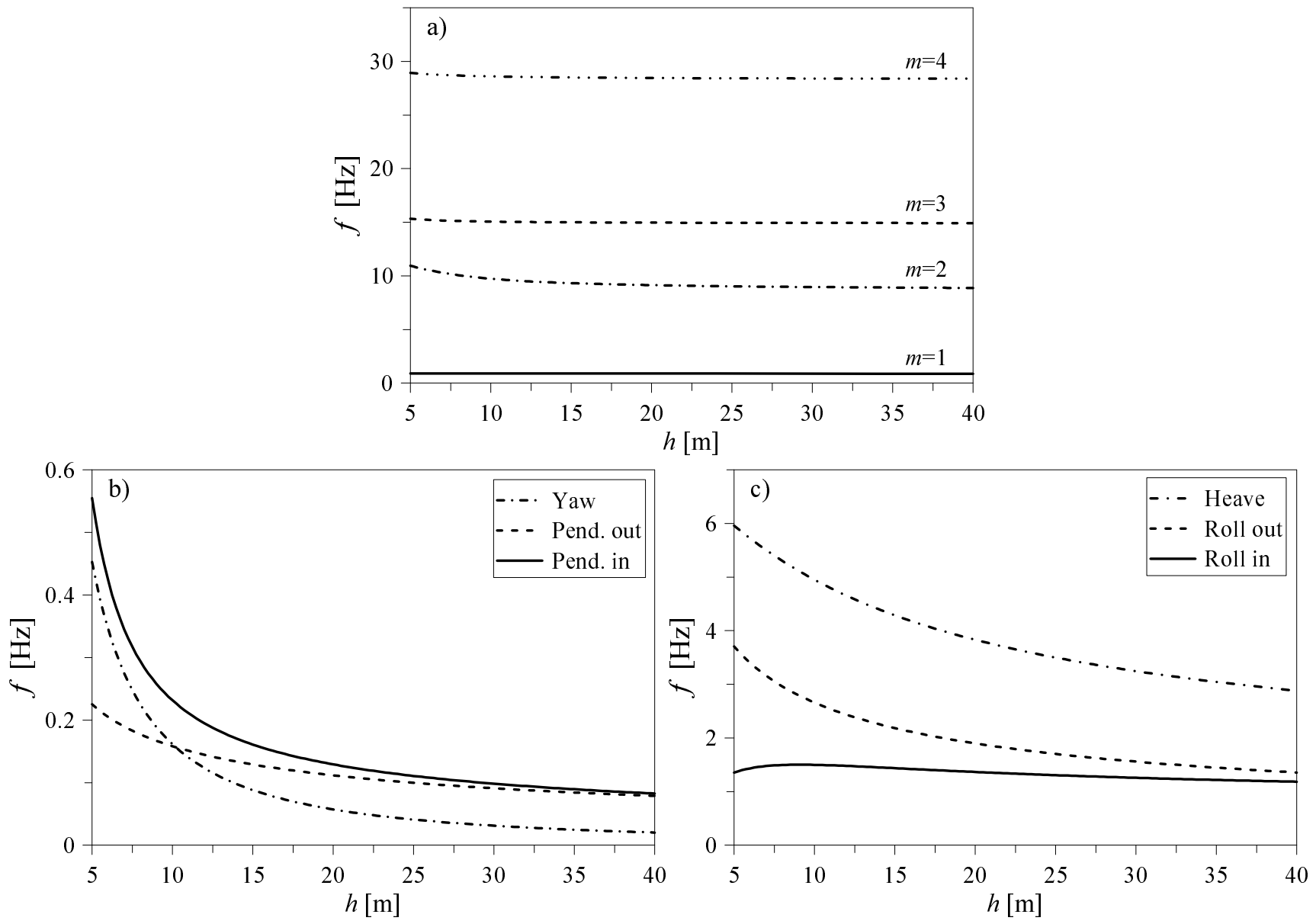

Figure 5: Container crane frequencies vs $h$ : a) boom lowest four frequencies $(m=1, \ldots, 4), b)$ pendulum in-plane mode (solid line), pendulum out-of-plane mode (dashed line), yaw mode (dasheddotted line), c) roll in-plane mode (solid line), roll out-of-plane mode (dashed line), heave mode (dashed-dotted line).

The container crane system behaves like a double pendulum in the planes $\left(\boldsymbol{e}_{1}, \boldsymbol{e}_{2}\right)$ and $\left(\boldsymbol{e}_{3}, \boldsymbol{e}_{1}\right)$ and like an elastic oscillator for the degrees of freedom $\bar{v}_{m}(t)\left(m=1, \ldots, N_{m}\right), l(t)$ and $\phi_{1}(t)$. The modes of the continuum-discrete system feature a coupling between the degrees of freedom of the boom and those of the container. Depending on the ratio between the modal components, the eigenvectors involving coordinates $\psi_{2}(t)$ and $\phi_{2}(t)$ are here classified as inplane pendulum mode and roll mode, respectively, whereas those involving coordinates $\psi_{3}(t)$ and $\phi_{3}(t)$ are the corresponding out-of-plane pendulum mode and roll mode, respectively. On the other hand, the modes related to $\phi_{1}(t)$ and $l(t)$ are classified respectively as the yaw mode and the heave mode, while the eigenvectors featuring $\bar{v}_{m}(t)\left(m=1, \ldots, N_{m}\right)$ with dominant participation are referred to as boom modes. The first investigation on the modal properties 
of the system is carried out assuming the container positioned at the boom tip $\left(x_{c}=L\right)$ with its full payload (see Table 2).

Figures 5a-c show variation of the container crane eigenfrequencies increasing with the height $h$ of the container relative to the boom. The frequencies of the lowest four modes of the boom are reported in Fig. 5a from which, as expected, it is apparent that these frequencies are not influenced by the parameter $h$ and that only the first mode possesses a frequency (about $0.9 \mathrm{~Hz}$ in the whole range of $h$ ) close to that of the container frequencies. The latter are reported in Fig. 5b for the yaw and pendular modes and in Fig. 5c for the heave and roll modes, respectively.

Figure 6 contrasts the frequencies of the container modes obtained by neglecting or considering the boom deformability. The boom deformability has negligible influence on the pendular modes, in particular, the in-plane mode is slightly affected by the boom elasticity in a small range of $h(h=5 \mathrm{~m}$ to $15 \mathrm{~m})$ whereas the out-of-plane mode, operating at a lower frequency, is barely affected by the crane deformability. Finally, the yaw, heave, and roll modes, which involve the elongation of the hoisting cables and, as such, the vertical boom displacement, show frequencies which can be $20-30 \%$ (to $55 \%$ in the case of the out-of-plane roll mode) lower than those possessed by the crane model with undeformable boom.
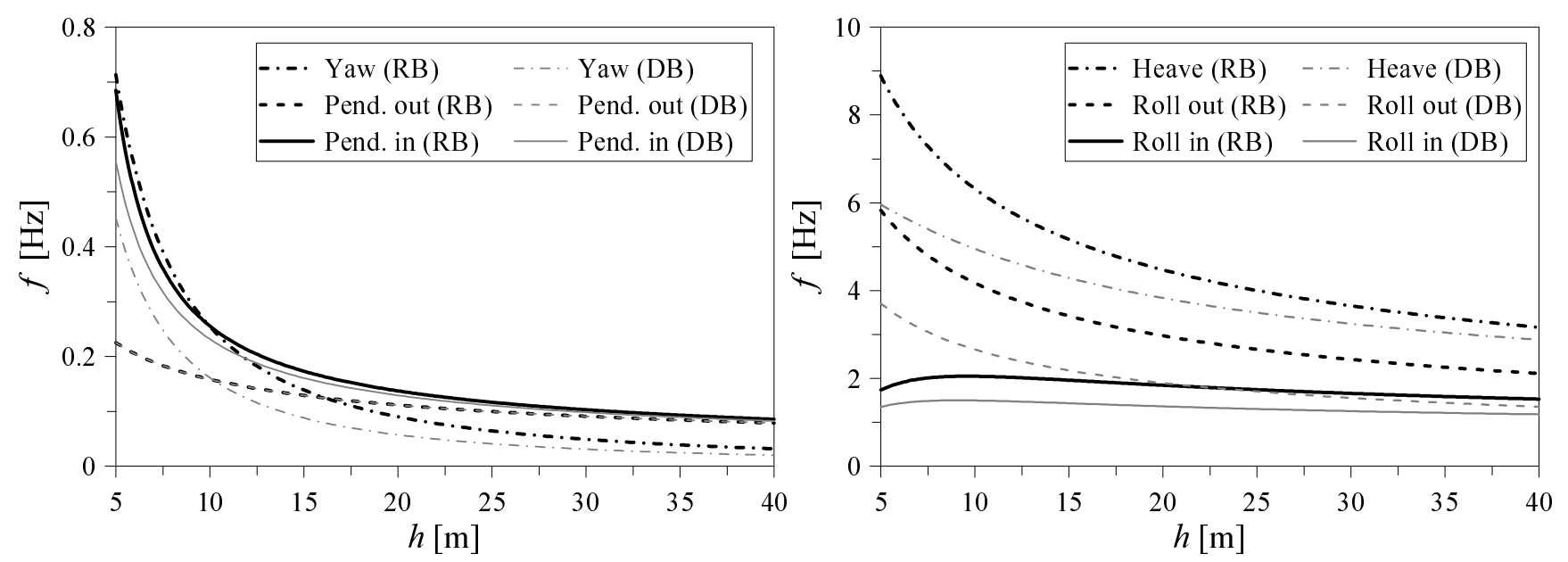

Figure 6: Container frequencies vs $h$ : (left) pendulum in-plane mode (solid line), pendulum out-ofplane mode (dashed line), yaw mode (dashed-dotted line), (right) roll in-plane mode (solid line), roll out-of-plane mode (dashed line), heave mode (dashed-dotted line). Comparison between rigid (RB) (black lines) and deformable (DB) (grey lines) boom models. 


\subsection{Onset of autoparametric resonances}

As known $[17,16]$, when the frequencies of two or more modes are in a suitable integer ratio (1:1, 2:1, 3:1, etc.), internal (autoparametric) resonances may occur between the corresponding modes. Thus, for certain initial conditions or excitations injecting energy into one of these modes, the energy can be transferred to the autoparametrically coupled modes giving rise to undesired coupled-mode motions. This is indeed a dynamic instability of the considered equilibrium state of the crane multibody system.

To highlight conditions for the onset of such resonances, the evaluation of the ratio between the frequencies of the container modes and those of the boom modes is a necessary step. In particular, specific attention is paid to potential interactions between the lowest boom mode (possessing a frequency close to those of the container) and the in-plane and out-ofplane pendular modes. Since the modal characteristics of the container crane vary depending on the inertial and geometric configurations of the multibody system, parametric analyses are performed to evaluate the ratio between the above mentioned modes for several crane configurations. Figure 7a shows these frequency ratios varying the length of the hoisting cables (height $h$ ) with the container positioned at the boom tip $\left(x_{c}=L\right)$. Two payloads are considered, namely, the minimum and maximum allowable payload. As shown in Fig. 7a, the boom lowest flexural frequency and the out-of-plane pendular frequency can be in ratios higher than $4: 1$, representing higher-order resonances devoid of practical meaning. On the other hand, $2: 1$ and $3: 1$ ratios can take place among the boom lowest frequency and the in-plane pendular frequency. In particular, a range of $h$ giving rise to $2: 1$ and $3: 1$ ratios occurs when the container has its maximum payload due to the fact that, although the pendular mode is not influenced by the mass, the boom flexural modes strongly depend on the lumped mass which induces a decrease of the corresponding flexural frequencies. Moreover, as highlighted in Fig. 7b, by moving the container along the boom (i.e., by increasing the trolley distance from the boom tip), the modal mass contribution of the container in the flexural motions decreases and thus the ratio between the boom lowest frequency and that of the pendular modes (which does not vary with the container position along the boom) increases.

In conclusion, the sensitivity analyses carried out to study the influence of the main geometric and mechanical parameters of the container crane system on the modal behaviour allows determination of the crane configurations in which $2: 1$ or $3: 1$ nonlinear interactions can take place between the boom bending mode and the container in-plane pendular mode. 

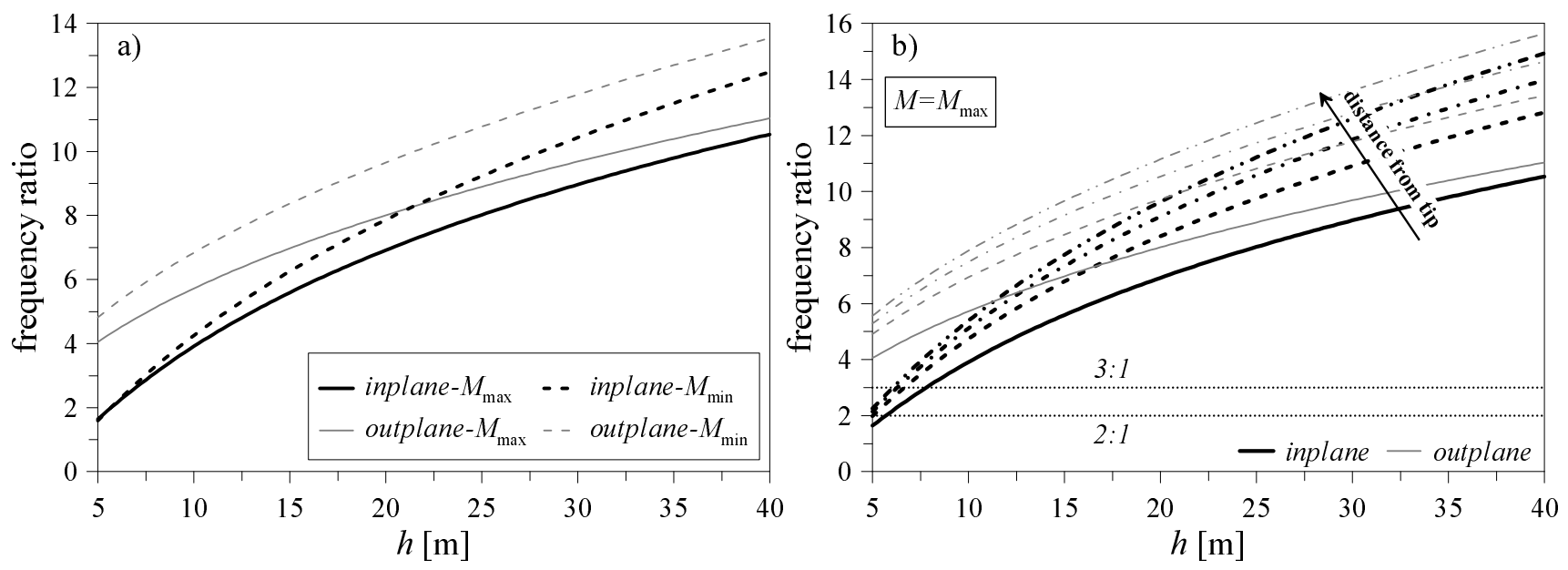

Figure 7: Frequency ratios of the in-plane and out-of-plane pendular modes and the lowest boom mode vs. $h$. (a) The container is positioned at the boom tip $\left(x_{c}=L\right)$, (b) the container positions are varied along the boom considering the maximum container size $\left(M=M_{\max }\right)$.

This happens when the container is positioned high, close to the boom tip. The corresponding range is $h=5 \mathrm{~m}$ to $8 \mathrm{~m}$. This may occur before the descending phase of the bell representing a typical trajectory during cargo loading on the cargo ship. This configuration is adopted next to investigate the nonlinear 2:1 interaction between the boom and the container modes. To this end, the method of multiple scales $[17,18,16]$ is employed together with pathfollowing tools for continuation analysis of the solutions of the modulation equations. Ranges of stability/instability of the single-mode and 2:1 resonant coupled-mode responses will be highlighted together with the relevant bifurcations.

\section{Perturbation Analysis}

The method of multiple scales $[17,18,16]$ is here employed to perform the perturbation analysis of the equations of motion governing the dynamics of the container crane system. Due to the fact that the 2:1 internal resonance involves the lowest boom bending mode and the in-plane pendular mode, the resonant dynamics take place in the vertical plane $\left(\boldsymbol{e}_{3}, \boldsymbol{e}_{1}\right)$ thus allowing reduction of the mechanical model to a planar model. Consequently, the kinematics of the system are restricted to the subset of four generalized coordinates $\xi(t)=\left\{\bar{v}_{1}(t), l(t), \psi_{2}(t), \phi_{2}(t)\right\}$ assuming only one mode in the Faedo-Galerkin discretisation procedure (i.e., $N_{m}=1$ ). The 
ensuing reduced equations of motion read

$$
\frac{d}{d t}\left(\mathcal{L}_{\dot{\xi}_{k}}\right)-\mathcal{L}_{\xi_{k}}+d_{k} \dot{\xi}_{k}=Q_{k} \quad(k=1, \ldots, 4)
$$

The system is subject to a harmonic direct excitation given in the form $F(t)=A_{F} \cos (\Omega t)$ so that the right-end side term $Q_{k}$ in Eq. (15) represents the $k$ th Lagrangian component of the excitation.

Nondimensionalization. Equation (15) can be cast in nondimensional form adopting the total boom length $L$ and the characteristic frequency $\bar{f}=\sqrt{\frac{E J}{\rho A L^{4}}}$ as scaling parameters. Thus, the nondimensional Lagrangian coordinates, collected in the $4 \times 1$ column vector $\mathbf{x}=$ $\left[\begin{array}{llll}\mathrm{x}_{1} & \mathrm{x}_{2} & \mathrm{x}_{3} & \mathrm{x}_{4}\end{array}\right]^{\top}$, become

$$
\mathrm{x}_{1}=\frac{\bar{v}_{1}(t)}{L}, \mathrm{x}_{2}=\frac{l(t)}{L}, \mathrm{x}_{3}=\psi_{2}(t), \mathrm{x}_{4}=\phi_{2}(t),
$$

while, by introducing the nondimensional time $\bar{t}=\bar{f} t$, the nondimensional time rates are expressed as

$$
\dot{\mathrm{x}}_{1}=\frac{1}{\bar{f} L} \frac{d \bar{v}_{1}(t)}{d t}, \dot{\mathrm{x}}_{2}=\frac{1}{\bar{f} L} \frac{d l(t)}{d t}, \dot{\mathrm{x}}_{3}=\frac{1}{\bar{f}} \frac{d \psi_{2}(t)}{d t}, \dot{\mathrm{x}}_{4}=\frac{1}{\bar{f}} \frac{d \phi_{2}(t)}{d t},
$$

where the overdot indicates, here and henceforth, differentiation with respect to the nondimensional time. Moreover, assuming the amplitude of the external excitation $F(t)$ proportional to the total weight of the crane boom yields $F(t)=\lambda(\rho A g L) \cos \Omega t$ which, in nondimensional form, becomes $\bar{F}(\bar{t})=c_{f} \lambda \cos \bar{\Omega} \bar{t}$ with $c_{f}=g /\left(L \bar{f}^{2}\right)$ and $\bar{\Omega}=\Omega / \bar{f}$.

State-space representation. Equations of motion (15), cast in nondimensional form and not explicitly reported here for the sake of conciseness, represent a set of four fully nonlinear ordinary differential equations. To obtain asymptotic expansions of the solutions of Eqs. (15) by the method of multiple scales, the equations of motion are Taylor expanded up to third order. The thus reduced four-dof system is described by the following nondimensional vectorvalued equations of motion [10]:

$$
\begin{aligned}
& \mathbf{M}_{1} \ddot{\mathbf{x}}+\mathbf{C}_{1} \dot{\mathbf{x}}+\mathbf{K}_{1} \mathbf{x} \\
& +\mathbf{M}_{2}(\dot{\mathbf{x}}, \dot{\mathbf{x}})+\mathbf{M}_{2}^{*}(\mathbf{x}, \ddot{\mathbf{x}})+\mathbf{K}_{2}(\mathbf{x}, \mathbf{x}) \\
& +\mathbf{M}_{3}(\mathbf{x}, \dot{\mathbf{x}}, \dot{\mathbf{x}})+\mathbf{M}_{3}^{*}(\mathbf{x}, \mathbf{x}, \ddot{\mathbf{x}})+\mathbf{K}_{3}(\mathbf{x}, \mathbf{x}, \mathbf{x})+\lambda \cos \Omega t \mathbf{F}=0
\end{aligned}
$$

where the overbar denoting nondimensional variables and parameters, here and henceforth, is dropped for the sake of notational simplicity. In Eq. (18), $\mathbf{M}_{1}, \mathbf{C}_{1}$ and $\mathbf{K}_{1}$ are $4 \times 4$ symmetric 
and positive-defined matrices representing the linear mass, damping and stiffness operators, respectively; $\mathbf{M}_{2}, \mathbf{M}_{2}^{*}, \mathbf{M}_{3}, \mathbf{M}_{3}^{*}$ are $4 \times 1$ noncommutative and multilinear operators which represent the quadratic and cubic parts of the nonlinear inertia, respectively, whereas $\mathbf{K}_{2}$ and $\mathbf{K}_{3}$ are analogous operators representing the quadratic and cubic parts of the nonlinear stiffness. Finally, $\mathbf{F}$ is a $4 \times 1$ vector collecting the amplitude of the external excitation. Let $\boldsymbol{\Psi}$ represent the linear modal tensor such that, introducing the transformation $\mathbf{x}=\mathbf{\Psi} \mathbf{q}$, the set of equations (18) can be rewritten in normal coordinates $\mathrm{q}_{j}$ as

$$
\begin{aligned}
\ddot{\mathbf{q}}+\Lambda \mathbf{q}= & -\mathbf{Z} \dot{\mathbf{q}}-\mathbf{G}_{2}(\mathbf{q}, \mathbf{q})-\mathbf{I}_{2}(\dot{\mathbf{q}}, \dot{\mathbf{q}})-\mathbf{I}_{2}^{*}(\mathbf{q}, \ddot{\mathbf{q}}) \\
& -\mathbf{G}_{3}(\mathbf{q}, \mathbf{q}, \mathbf{q})-\mathbf{I}_{3}(\mathbf{q}, \dot{\mathbf{q}}, \dot{\mathbf{q}})-\mathbf{I}_{3}^{*}(\mathbf{q}, \mathbf{q}, \ddot{\mathbf{q}})-\lambda \cos \Omega t \mathbf{P}
\end{aligned}
$$

where $\boldsymbol{\Lambda}=\boldsymbol{\Psi}^{\top} \mathbf{K}_{1} \boldsymbol{\Psi}=\omega_{i}^{2} \delta_{i j}$ is the diagonal matrix collecting the square of the system eigenfrequencies and $\boldsymbol{\Psi}$ is such that $\boldsymbol{\Psi}^{\top} \mathbf{M}_{1} \boldsymbol{\Psi}=\mathbf{I}$ with $\mathbf{I}=\delta_{i j}$ and $\delta_{i j}$ being the Kronecker delta. Moreover, $\mathbf{Z}=\boldsymbol{\Psi}^{\top} \mathbf{C}_{1} \boldsymbol{\Psi}$ is the diagonal matrix of the linear modal damping, $\mathbf{P}=\boldsymbol{\Psi}^{\top} \mathbf{F}$ is the modal projection of the external excitation, and the operators appearing in the right-end side are expressed as

$$
\begin{aligned}
& \mathbf{G}_{2}(\mathbf{q}, \mathbf{q})=\Psi^{\top} \mathbf{K}_{2}(\Psi \mathbf{q}, \Psi \mathbf{q}), \mathbf{G}_{3}(\mathbf{q}, \mathbf{q}, \mathbf{q})=\Psi^{\top} \mathbf{K}_{3}(\Psi \mathbf{q}, \Psi \mathbf{q}, \Psi \mathbf{q}) \\
& \mathbf{I}_{2}(\dot{\mathbf{q}}, \dot{\mathbf{q}})=\Psi^{\top} \mathbf{M}_{2}(\Psi \dot{\mathbf{q}}, \Psi \dot{\mathbf{q}}), \mathbf{I}_{3}(\mathbf{q}, \dot{\mathbf{q}}, \dot{\mathbf{q}})=\Psi^{\top} \mathbf{M}_{3}(\Psi \mathbf{q}, \Psi \dot{\mathbf{q}}, \Psi \dot{\mathbf{q}}) \\
& \mathbf{I}_{2}^{*}(\mathbf{q}, \ddot{\mathbf{q}})=\Psi^{\top} \mathbf{M}_{2}^{*}(\Psi \mathbf{q}, \Psi \ddot{\mathbf{q}}), \mathbf{I}_{3}^{*}(\mathbf{q}, \mathbf{q}, \ddot{\mathbf{q}})=\Psi^{\top} \mathbf{M}_{3}^{*}(\Psi \mathbf{q}, \Psi \mathbf{q}, \Psi \ddot{\mathbf{q}}) .
\end{aligned}
$$

The second order (in time) set of equations (19) is conveniently reduced to a first-order system (state-space representation) as follows:

$$
\begin{aligned}
\dot{\mathbf{q}}-\mathbf{p}= & \mathbf{0} \\
\dot{\mathbf{p}}+\mathbf{\Lambda} \mathbf{q}= & -\mathbf{Z} \mathbf{p}-\mathbf{G}_{2}(\mathbf{q}, \mathbf{q})-\mathbf{I}_{2}(\mathbf{p}, \mathbf{p})-\mathbf{I}_{2}^{*}(\mathbf{q}, \dot{\mathbf{p}}) \\
& -\mathbf{G}_{3}(\mathbf{q}, \mathbf{q}, \mathbf{q})-\mathbf{I}_{3}(\mathbf{q}, \mathbf{p}, \mathbf{p})-\mathbf{I}_{3}^{*}(\mathbf{q}, \mathbf{q}, \dot{\mathbf{p}})-\lambda \cos \Omega t \mathbf{P}
\end{aligned}
$$

By letting $\epsilon$ denote a small nondimensional parameter representing the norm of the deviation of the current crane configuration form its reference state, a third-order expansion of the solutions of (21) is sought in the form

$$
\mathbf{q}=\sum_{i=1}^{3} \epsilon^{i} \mathbf{q}_{i}\left(T_{0}, T_{1}, T_{2}\right), \mathbf{p}=\sum_{i=1}^{3} \epsilon^{i} \mathbf{p}_{i}\left(T_{0}, T_{1}, T_{2}\right)
$$

where $T_{0}=t$ is the nondimensional fast time scale and $T_{1}=\epsilon t$ and $T_{2}=\epsilon^{2} t$ are the nondimensional slow time scales in terms of which the nondimensional time derivative is $d / d t=$ $D_{0}+\epsilon D_{1}+\epsilon^{2} D_{2}+\ldots$ with $D_{j}=\partial / \partial T_{j}(j=0,1,2)$. 
The perturbation analysis will be carried out studying, first, the effects of quadratic nonlinearities, thus only terms up to order $\epsilon^{2}$ will be retained. Subsequently, cubic nonlinearities will be added enriching the asymptotic treatment to include the perturbation problems up to order $\epsilon^{3}$.

\subsection{Two-to-one internal resonance: second order approxima- tion}

The perturbation treatment requires a preliminary rescaling of the damping force and excitation with the guiding principle that the internal/external resonant forces, the inertia and the damping forces should balance each other. The linear damping force is assumed to be a second order effect which, analytically, implies a re-scaling of $\mathbf{C}_{1}$ as $\epsilon \mathbf{C}_{1}$ in Eq. (18) thus making the damping forces appear at second order in Eq. (21). Also the forcing term is rescaled to appear at second order, namely, $\lambda=\epsilon^{2} \lambda$. By substituting (22) into (21), the following hierarchy of linear problems is obtained by equating to zero coefficients of like powers of $\epsilon$ :

Order $\epsilon$ :

$$
\begin{aligned}
& D_{0} \mathbf{q}_{1}-\mathbf{p}_{1}=\mathbf{0} \\
& D_{0} \mathbf{p}_{1}+\boldsymbol{\Lambda} \mathbf{q}_{1}=\mathbf{0}
\end{aligned}
$$

Order $\epsilon^{2}$ :

$$
\begin{aligned}
& D_{0} \mathbf{q}_{2}-\mathbf{p}_{2}=-D_{1} \mathbf{q}_{1} \\
& D_{0} \mathbf{p}_{2}+\boldsymbol{\Lambda} \mathbf{q}_{2}=-D_{1} \mathbf{p}_{1}-\mathbf{Z} \mathbf{p}_{1}-\mathbf{G}_{2}\left(\mathbf{q}_{1}, \mathbf{q}_{1}\right)-\mathbf{I}_{2}\left(\mathbf{p}_{1}, \mathbf{p}_{1}\right)-\mathbf{I}_{2}^{*}\left(\mathbf{q}_{1}, D_{0} \mathbf{p}_{1}\right)-\lambda \cos \Omega T_{0} \mathbf{P}
\end{aligned}
$$

Since two modes only are involved in the 2:1 internal resonance, the general solution of Eq. (23) can be expressed as

$$
\begin{aligned}
& \mathbf{q}_{1}=A_{m}\left(T_{1}, T_{2}\right) e^{i \omega_{m} T_{0}} \mathbf{u}_{m}+A_{n}\left(T_{1}, T_{2}\right) e^{i \omega_{n} T_{0}} \mathbf{u}_{n}+c c \\
& \mathbf{p}_{1}=i \omega_{m} A_{m}\left(T_{1}, T_{2}\right) e^{i \omega_{m} T_{0}} \mathbf{u}_{m}+i \omega_{n} A_{n}\left(T_{1}, T_{2}\right) e^{i \omega_{n} T_{0}} \mathbf{u}_{n}+c c
\end{aligned}
$$

where $\mathbf{u}_{m}$ and $\mathbf{u}_{n}$ are the eigenvectors of the two resonant linear normal modes of the system (in terms of modal coordinates, obtained as eigensolutions of Eq. (21) by setting its rightside to zero), $\omega_{m}$ and $\omega_{n}$ are the associated nondimensional eigenfrequencies, $A_{m}\left(T_{1}, T_{2}\right)$ and $A_{n}\left(T_{1}, T_{2}\right)$ are the unknown complex-valued amplitudes of the $m$ th and the $n$th modes and $c c$ stands for complex conjugate. By substituting Eq. (25) into Eq. (24) the $\epsilon^{2}$ order system reads

$$
\begin{aligned}
& D_{0} \mathbf{q}_{2}-\mathbf{p}_{2}=\mathbf{g}_{21}\left(A_{m}, A_{n} ; \omega_{m}, \omega_{n}\right)+c c \\
& D_{0} \mathbf{p}_{2}+\boldsymbol{\Lambda} \mathbf{q}_{2}=\mathbf{g}_{22}\left(A_{m}, A_{n} ; \omega_{m}, \omega_{n}\right)+c c
\end{aligned}
$$


The system (26) exhibits secular terms due to the primary resonance of the external excitation with the $n$th mode (expressed as $\Omega=\omega_{n}+\epsilon \sigma_{1}$, with $\sigma_{1}$ being a detuning parameter) and the autoparametric resonant terms caused by the $2: 1$ frequency ratio, $\omega_{n} \approx 2 \omega_{m}$. To remove the singularity, solvability conditions must be enforced. By introducing the solutions of the adjoint homogeneous problem of Eq. (26) in the form:

$$
\begin{aligned}
& \mathbf{q}_{m}^{*}=i \omega_{m} e^{-i \omega_{m} T_{0}} \mathbf{u}_{m}+c c, \mathbf{p}_{m}^{*}=e^{-i \omega_{m} T_{0}} \mathbf{u}_{m}+c c \\
& \mathbf{q}_{n}^{*}=i \omega_{n} e^{-i \omega_{n} T_{0}} \mathbf{u}_{n}+c c, \mathbf{p}_{n}^{*}=e^{-i \omega_{n} T_{0}} \mathbf{u}_{n}+c c
\end{aligned}
$$

the solvability of Eq. (26) is enforced requiring the orthogonality between (27) and (28) and the inhomogeneous term (right-end side) in Eq. (26). That is,

$$
\frac{\omega_{m}}{2 \pi} \int_{0}^{\frac{2 \pi}{\omega_{m}}}\left[\begin{array}{ll}
\mathbf{q}_{m}^{*} & \mathbf{p}_{m}^{*}
\end{array}\right]\left[\begin{array}{l}
\mathbf{g}_{21} \\
\mathbf{g}_{22}
\end{array}\right] d T_{0}=0, \frac{\omega_{n}}{2 \pi} \int_{0}^{\frac{2 \pi}{\omega_{n}}}\left[\begin{array}{ll}
\mathbf{q}_{n}^{*} & \mathbf{p}_{n}^{*}
\end{array}\right]\left[\begin{array}{l}
\mathbf{g}_{21} \\
\mathbf{g}_{22}
\end{array}\right] d T_{0}=0
$$

To express the closeness of the internal resonance, we let $\omega_{n}=2 \omega_{m}+\epsilon \sigma_{2}$, with $\sigma_{2}$ being a further detuning parameter. Moreover, to make use of complex algebra, let $\cos \Omega T_{0}=$ $\frac{1}{2} e^{i\left(T_{0} \omega_{n}+T_{1} \sigma_{1}\right)}+c c$. Thus substituting the external and internal resonance conditions into the solvability conditions (29), the following modulation equations for the amplitudes $A_{m}$ and $A_{n}$ are obtained:

$$
\begin{aligned}
& -2 i \omega_{m}\left(D_{1} A_{m}+\mu_{m} A_{m}\right)+\left(\alpha_{m_{1}}+\alpha_{m_{2}} \omega_{m}^{2}\right) e^{i T_{1} \sigma_{2}} \bar{A}_{m} A_{n}=0 \\
& -2 i \omega_{n}\left(D_{1} A_{n}+\mu_{n} A_{n}\right)+\left(\alpha_{n_{1}}+\alpha_{n_{2}} \omega_{n}^{2}\right) e^{-i T_{1} \sigma_{2}} A_{m}^{2}+\frac{1}{2} \lambda f_{n} e^{i T_{1} \sigma_{1}}=0
\end{aligned}
$$

where $\bar{A}_{m}$ is the conjugate of $A_{m}\left(\bar{A}_{n}\right.$ is the conjugate of $\left.A_{n}\right)$. The coefficients of Eq. (30) represent the coefficients of the quadratic nonlinear stiffness terms $\left(\alpha_{m_{1}}<0, \alpha_{n_{1}}<0\right)$, the quadratic nonlinear inertial terms $\left(\alpha_{m_{2}}<0, \alpha_{n_{2}}<0\right)$, the linear damping $\left(\mu_{m}>0, \mu_{n}>0\right)$ and the modal projection of the forcing term $\left(f_{n}=\mathbf{u}_{n}^{\top} \mathbf{F}<0\right)$.

By expressing the complex amplitudes $A_{m}, \bar{A}_{m}, A_{n}$ and $\bar{A}_{n}$ in polar form

$$
A_{m}\left(T_{1}, T_{2}\right)=\frac{1}{2} a_{m}\left(T_{1}, T_{2}\right) e^{i \theta_{m}\left(T_{1}, T_{2}\right)}, \quad A_{n}\left(T_{1}, T_{2}\right)=\frac{1}{2} a_{n}\left(T_{1}, T_{2}\right) e^{i \theta_{n}\left(T_{1}, T_{2}\right)}
$$

and substituting the polar form into Eq. (30), separating real and imaginary parts yield the following four differential equations governing the slow modulation of the modes amplitudes 
and relative phases:

$$
\begin{aligned}
& D_{1} a_{m}=-\mu_{m} a_{m}+\frac{1}{4 \omega_{m}}\left(\alpha_{m_{1}}+\alpha_{m_{2}} \omega_{m}^{2}\right) a_{m} a_{n} \sin \beta_{2} \\
& D_{1} a_{n}=-\mu_{n} a_{n}-\frac{1}{4 \omega_{n}}\left(\alpha_{n_{1}}+\alpha_{n_{2}} \omega_{n}^{2}\right) a_{m}^{2} \sin \beta_{2}+\frac{\lambda f_{n}}{2 \omega_{n}} \sin \beta_{1} \\
& D_{1} \beta_{1}=\sigma_{1}+\frac{1}{4 \omega_{n} a_{n}}\left(\alpha_{n_{1}}+\alpha_{n_{2}} \omega_{n}^{2}\right) a_{m}^{2} \cos \beta_{2}+\frac{\lambda f_{n}}{2 \omega_{n} a_{n}} \cos \beta_{1} \\
& D_{1} \beta_{2}=\sigma_{2}-\frac{1}{4 \omega_{n} a_{n}}\left(\alpha_{n_{1}}+\alpha_{n_{2}} \omega_{n}^{2}\right) a_{m}^{2} \cos \beta_{2}-\frac{\lambda f_{n}}{2 \omega_{n} a_{n}} \cos \beta_{1}+\frac{1}{2 \omega_{m}}\left(\alpha_{m_{1}}+\alpha_{m_{2}} \omega_{m}^{2}\right) a_{n} \cos \beta_{2}
\end{aligned}
$$

where $\beta_{1}\left(T_{1}, T_{2}\right)=\sigma_{1} T_{1}-\theta_{n}\left(T_{1}, T_{2}\right)$ and $\beta_{2}\left(T_{1}, T_{2}\right)=\sigma_{2} T_{1}-2 \theta_{m}\left(T_{1}, T_{2}\right)+\theta_{n}\left(T_{1}, T_{2}\right)$ are the relative phases.

\subsection{Third order approximation}

To study the effects of cubic nonlinearities, after substituting (22) into (21) and rescaling the terms $\mathbf{Z} \mathbf{p}$ and $\lambda$ to appear at order $\epsilon^{3}$, a hierarchy of linear problems is obtained where the first perturbation problem is Eq. (23), while the second and third perturbations are given by

Order $\epsilon^{2}$ :

$$
\begin{aligned}
& D_{0} \mathbf{q}_{2}-\mathbf{p}_{2}=-D_{1} \mathbf{q}_{1} \\
& D_{0} \mathbf{p}_{2}+\boldsymbol{\Lambda} \mathbf{q}_{2}=-D_{1} \mathbf{p}_{1}-\mathbf{G}_{2}\left(\mathbf{q}_{1}, \mathbf{q}_{1}\right)-\mathbf{I}_{2}\left(\mathbf{p}_{1}, \mathbf{p}_{1}\right)-\mathbf{I}_{2}^{*}\left(\mathbf{q}_{1}, D_{0} \mathbf{p}_{1}\right)
\end{aligned}
$$

Order $\epsilon^{3}$ :

$$
\begin{aligned}
& D_{0} \mathbf{q}_{3}-\mathbf{p}_{3}=-D_{1} \mathbf{q}_{2}-D_{2} \mathbf{q}_{1} \\
& D_{0} \mathbf{p}_{3}+\boldsymbol{\Lambda} \mathbf{q}_{3}=-D_{1} \mathbf{p}_{2}-D_{2} \mathbf{p}_{1}-\mathbf{Z} \mathbf{p}_{1}-\mathbf{G}_{2}\left(\mathbf{q}_{1}, \mathbf{q}_{2}\right)-\mathbf{I}_{2}\left(\mathbf{p}_{1}, \mathbf{p}_{2}\right)-\mathbf{I}_{21}^{*}\left(\mathbf{q}_{2}, D_{0} \mathbf{p}_{1}\right)-\mathbf{I}_{22}^{*}\left(\mathbf{q}_{1}, D_{0} \mathbf{p}_{2}\right) \\
& \quad-\mathbf{I}_{23}^{*}\left(\mathbf{q}_{1}, D_{1} \mathbf{p}_{1}\right)-\mathbf{G}_{3}\left(\mathbf{q}_{1}, \mathbf{q}_{1}, \mathbf{q}_{1}\right)-\mathbf{I}_{3}\left(\mathbf{q}_{1}, \mathbf{p}_{1}, \mathbf{p}_{1}\right)-\mathbf{I}_{3}^{*}\left(\mathbf{q}_{1}, \mathbf{q}_{1}, D_{0} \mathbf{p}_{1}\right)-\lambda \cos \Omega T_{0} \mathbf{P}
\end{aligned}
$$

where $\mathbf{I}_{21}^{*}\left(\mathbf{q}_{2}, D_{0} \mathbf{p}_{1}\right), \mathbf{I}_{22}^{*}\left(\mathbf{q}_{1}, D_{0} \mathbf{p}_{2}\right), \mathbf{I}_{23}^{*}\left(\mathbf{q}_{1}, D_{1} \mathbf{p}_{1}\right)$ are inertial terms with quadratic nonlinearities ensuing from $\mathbf{I}_{2}^{*}(\mathbf{q}, \dot{\mathbf{p}})$.

The perturbation treatment up to second order follows the same steps shown in the previous section. However, to investigate the effects of the cubic nonlinearities in the two-to-one internal resonance, the external resonance condition is recast as $\Omega=\omega_{n}+\epsilon^{2} \sigma_{1}$ because the forcing term is of order $\epsilon^{3}$. The consequence is that $\lambda \cos \Omega T_{0}=\frac{1}{2} \lambda e^{i\left(T_{0} \omega_{n}+\sigma_{1} T_{2}\right)}+c c$.

Substituting Eq. (25) into Eq. (33) and enforcing the orthogonality of the inhomogeneous terms (right-hand side of Eq. (33)) to the solutions of the adjoint homogeneous problem (i.e., 
solvability condition) yield the following modulation equations:

$$
\begin{aligned}
& -2 i \omega_{m} D_{1} A_{m}+\left(\alpha_{m_{1}}+\alpha_{m_{2}} \omega_{m}^{2}\right) e^{i T_{1} \sigma_{2}} \bar{A}_{m} A_{n}=0, \\
& -2 i \omega_{n} D_{1} A_{n}+\left(\alpha_{n_{1}}+\alpha_{n_{2}} \omega_{n}^{2}\right) e^{-i T_{1} \sigma_{2}} A_{m}^{2}=0,
\end{aligned}
$$

from which the rates (with respect to the slow scale $T_{1}$ ) of the complex-valued amplitudes, $D_{1} A_{m}$ and $D_{1} A_{n}$, are obtained.

By substituting the thus obtained amplitude rates into the second order problem given by (33), its solution can be sought in the form

$$
\begin{aligned}
\mathbf{q}_{2}= & \tilde{\mathbf{q}}_{1} A_{m}^{2} e^{2 i T_{0} \omega_{m}}+\tilde{\mathbf{q}}_{2} A_{n}^{2} e^{2 i T_{0} \omega_{n}}+\tilde{\mathbf{q}}_{3} A_{m} A_{n} e^{i T_{0}\left(\omega_{m}+\omega_{n}\right)} \\
& +\tilde{\mathbf{q}}_{4} \bar{A}_{m} A_{n} e^{i T_{0}\left(\omega_{n}-\omega_{m}\right)}+\tilde{\mathbf{q}}_{5} \bar{A}_{m} A_{m}+\tilde{\mathbf{q}}_{6} \bar{A}_{n} A_{n}+c c, \\
\mathbf{p}_{2}= & \tilde{\mathbf{p}}_{1} A_{m}^{2} e^{2 i T_{0} \omega_{m}}+\tilde{\mathbf{p}}_{2} A_{n}^{2} e^{2 i T_{0} \omega_{n}}+\tilde{\mathbf{p}}_{3} A_{m} A_{n} e^{i T_{0}\left(\omega_{m}+\omega_{n}\right)} \\
& +\tilde{\mathbf{p}}_{4} \bar{A}_{m} A_{n} e^{i T_{0}\left(\omega_{n}-\omega_{m}\right)}+\tilde{\mathbf{p}}_{5} \bar{A}_{m} A_{m}+\tilde{\mathbf{p}}_{6} \bar{A}_{n} A_{n}+c c,
\end{aligned}
$$

where $\tilde{\mathbf{q}}_{i}$ and $\tilde{\mathbf{p}}_{i}(i=1, \ldots, 6)+c c$ are the combination of the 4 eigenvectors $\mathbf{u}_{j}$ of the system

$$
\tilde{\mathbf{q}}_{i}=\sum_{j=1}^{4} \tilde{q}_{i, j} \mathbf{u}_{j}, \tilde{\mathbf{p}}_{i}=\sum_{j=1}^{4} \tilde{p}_{i, j} \mathbf{u}_{j} .
$$

Then, the solution (36) is substituted into the second order problem (33). Next, the orthogonality conditions are imposed between the solution of the adjoint homogeneous problem and the components of the second order solution resonant in $\omega_{m}$ and $\omega_{n}$, respectively; that is, the terms proportional to $e^{i T_{0} \omega_{m}}$ (i.e., $\left.\left(\tilde{\mathbf{q}}_{4}, \tilde{\mathbf{p}}_{4}\right)\right)$ and $e^{i T_{0} \omega_{n}}$ (i.e., $\left.\left(\tilde{\mathbf{q}}_{1}, \tilde{\mathbf{p}}_{1}\right)\right)$. Finally, by equating coefficients of like terms (i.e., having the same frequency) in the second order problem (33), coefficients $\tilde{q}_{i, j}$ and $\tilde{p}_{i, j}(i=1, \ldots, 6, j=1, \ldots, 4)$ can be determined. After obtaining the second order solution (36) and substituting it into the third order problem (34), the following inhomogeneous problem is obtained:

$$
\begin{aligned}
& D_{0} \mathbf{q}_{3}-\mathbf{p}_{3}=\mathbf{g}_{31}\left(A_{m}, A_{n} ; \omega_{m}, \omega_{n}\right)+c c \\
& D_{0} \mathbf{p}_{3}+\boldsymbol{\Lambda} \mathbf{q}_{3}=\mathbf{g}_{32}\left(A_{m}, A_{n} ; \omega_{m}, \omega_{n}\right)+c c .
\end{aligned}
$$

The solvability condition of (38) can be determined again by enforcing the orthogonality of $\left(\mathbf{g}_{31}, \mathbf{g}_{32}\right)$ to the solutions of the adjoint homogeneous problem

$$
\frac{\omega_{m}}{2 \pi} \int_{0}^{\frac{2 \pi}{\omega_{m}}}\left[\begin{array}{ll}
\mathbf{q}_{m}^{*} & \mathbf{p}_{m}^{*}
\end{array}\right]\left[\begin{array}{l}
\mathbf{g}_{31} \\
\mathbf{g}_{32}
\end{array}\right] d T_{0}=0, \frac{\omega_{n}}{2 \pi} \int_{0}^{\frac{2 \pi}{\omega_{n}}}\left[\begin{array}{ll}
\mathbf{q}_{n}^{*} & \mathbf{p}_{n}^{*}
\end{array}\right]\left[\begin{array}{l}
\mathbf{g}_{31} \\
\mathbf{g}_{32}
\end{array}\right] d T_{0}=0
$$

which yields the following modulation equations for the amplitudes $A_{m}$ and $A_{n}$ :

$$
\begin{aligned}
& -2 i \omega_{m}\left(D_{2} A_{m}+\mu_{m} A_{m}\right)+\alpha_{m_{3}}\left(\omega_{m}\right) \bar{A}_{m} A_{m}^{2}+\alpha_{m_{4}}\left(\omega_{m}\right) A_{m} \bar{A}_{n} A_{n}=0, \\
& -2 i \omega_{n}\left(D_{2} A_{n}+\mu_{n} A_{n}\right)+\alpha_{n_{3}} \bar{A}_{n} A_{n}^{2}+\alpha_{n_{4}}\left(\omega_{n}\right) \bar{A}_{m} A_{m} A_{n}+\frac{1}{2} f_{n} \lambda e^{i T_{2} \sigma_{1}}=0,
\end{aligned}
$$


where $\alpha_{n_{3}}>0$ and $\alpha_{m_{3}}\left(\omega_{m}\right), \alpha_{m_{4}}\left(\omega_{m}\right)$ and $\alpha_{n_{4}}\left(\omega_{n}\right)$ are nonlinear functions of the resonant frequencies $\omega_{m}$ and $\omega_{n}$, respectively. From Eq. (41) it is then possible to obtain the amplitudes rates (i.e., with respect to the slow time scale $T_{2}$ ), $D_{2} A_{m}$ and $D_{2} A_{n}$.

By using the method of reconstitution $[16,11]$, it is then possible to express the time rates of the modal amplitudes as $\dot{A}_{m}=\epsilon D_{1} A_{m}+\epsilon^{2} D_{2} A_{m}+\ldots, \dot{A}_{n}=\epsilon D_{1} A_{n}+\epsilon^{2} D_{2} A_{n}+\ldots$ and thus obtain the modulation equations up to third order as

$$
\begin{aligned}
2 i \omega_{m} \dot{A}_{m}= & -2 i \omega_{m} \mu_{m} A_{m}+\left(\alpha_{m_{1}}+\alpha_{m_{2}} \omega_{m}^{2}\right) e^{i \sigma_{2} t} \bar{A}_{m} A_{n} \\
& +\alpha_{m_{3}}\left(\omega_{m}\right) \bar{A}_{m} A_{m}^{2}+\alpha_{m_{4}}\left(\omega_{m}\right) A_{m} \bar{A}_{n} A_{n} \\
2 i \omega_{n} \dot{A}_{n}= & -2 i \omega_{n} \mu_{n} A_{n}+\left(\alpha_{n_{1}}+\alpha_{n_{2}} \omega_{n}^{2}\right) e^{-i \sigma_{2} t} A_{m}^{2} \\
& +\alpha_{n_{3}} \bar{A}_{n} A_{n}^{2}+\alpha_{n_{4}}\left(\omega_{n}\right) \bar{A}_{m} A_{m} A_{n}+\frac{1}{2} f_{n} \lambda e^{i \sigma_{1} t} .
\end{aligned}
$$

where the ordering parameter $\epsilon$ has been reabsorbed into the detuning parameters and amplitudes. Finally, introducing the polar form (31) of the complex amplitudes $A_{m}(t), \bar{A}_{m}(t)$, $A_{n}(t)$ and $\bar{A}_{n}(t)$, and substituting it into Eq. (41), separating real and imaginary parts yield the four differential equations governing the modulation of the (real) amplitudes and relative phases $\beta_{1}(t)=\sigma_{1} t-\theta_{n}(t)$ and $\beta_{2}(t)=\sigma_{2} t-2 \theta_{m}(t)+\theta_{n}(t)$.

\section{Bifurcation Analysis}

The aim of the bifurcation analysis carried out on the second and third order modulation equations is twofold: (i) to shed light onto the crane response regimes in the neighbourhood of the 2:1 internal resonance, (ii) to compare the scenarios predicted by the two expansions, the low and higher order expansions. We first discuss the results of the bifurcation analysis carried out on the second order modulation equations (32).

Second order bifurcation analysis. We consider the fixed points of the second order modulation equations (32), $D_{1} a_{m}=0, D_{1} a_{n}=0, D_{1} \beta_{1}=0$ and $D_{1} \beta_{2}=0$, which give rise to periodic solutions of the original crane system.

The ensuing system of four nonlinear algebraic equations in the unknowns $a_{m}, a_{n}, \beta_{1}$ and $\beta_{2}$ can be solved, for fixed values of the detuning parameters $\sigma_{1}$ and $\sigma_{2}$, in terms of the forcing amplitude $\lambda$ to obtain the force-response curve represented by thick solid lines in Fig. 8. The stability is ascertained by monitoring the eigenvalues of the Jacobian matrix of the equations system governing the slow dynamics of the amplitudes and phases. The obtained 


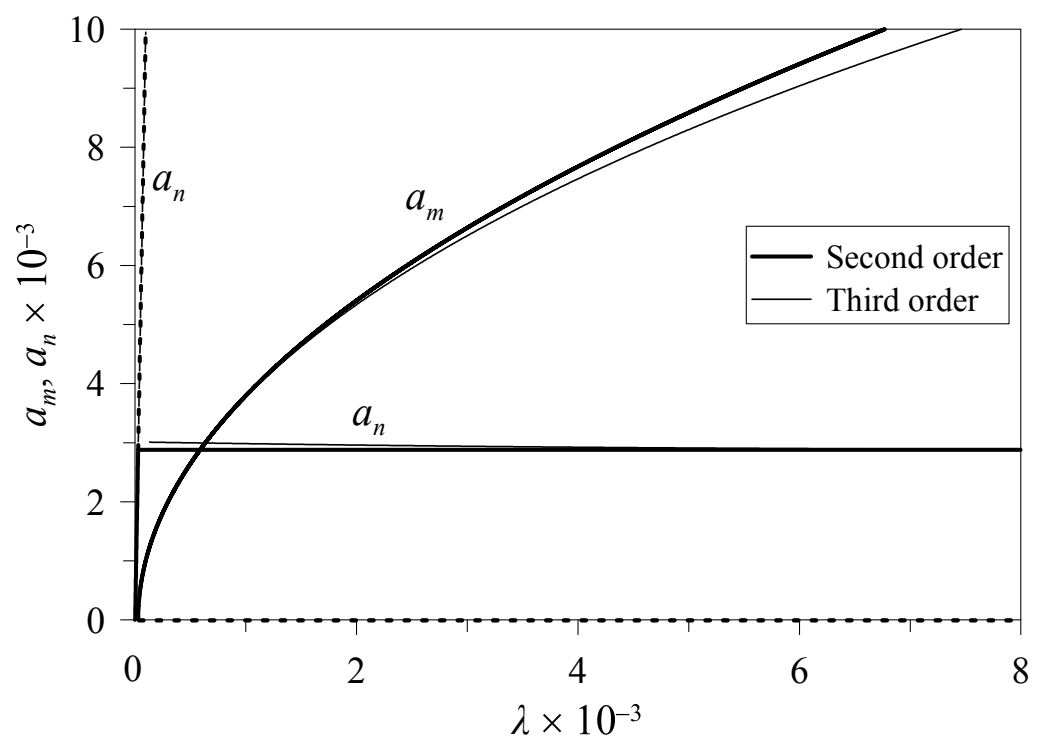

Figure 8: Force response curves for $\sigma_{1}=0$ and $\sigma_{2}=0$.

force-response curve shows a paradigmatic behaviour in the context of 2:1 internal resonances for a variety of nonlinear systems, the so-called saturation phenomenon. The response of the directly excited mode grows linearly up to a threshold excitation amplitude past which the single-mode response becomes unstable through a pitchfork bifurcation in favour of a two-mode response characterised by the fact that the directly excited response saturates at a constant amplitude while the autoparametrically excited mode grows nonlinearly with the force amplitude. In this figure we also show the bifurcation scenario (thin solid lines) predicted by the third order equations (41). The third order effects are such that past the pitchfork bifurcation, the amplitude of the saturated mode slightly decreases with the forcing amplitude instead of being constant as in the second order solution while the amplitude of the $m$ th mode increases at a slightly lower rate than that of the second order solution.

The bifurcation behaviour is further studied in terms of frequency-response curves obtained by pathfollowing (using a continuation code written in Mathematica by the authors) the fixed points of the modulation equations while monitoring the stability by simultaneously pathfollowing the eigenvalues of the Jacobian matrix.

The frequency-response curves are obtained by setting the forcing amplitude $\lambda$ and internal detuning $\sigma_{2}$ while selecting the external frequency detuning $\sigma_{1}$ (i.e., detuning between $\Omega$ and the frequency $\omega_{n}$ of the high-frequency mode) as control parameter. Two excitation levels are considered in the range where the autoparametric resonance is activated (i.e., coupled two-mode response), namely, a low level $\lambda=0.4 \times 10^{-3}$ and a higher level $\lambda=10^{-3}$. For a 
perfect tuning among the frequencies of the resonant modes $\left(\sigma_{2}=0\right)$, the frequency response curves are reported in Fig. 9 while the frequency-response curves obtained for a positive or a negative detuning $\sigma_{2}$ are shown in Fig. 10. The first observation is that these curves enjoy a full symmetry with respect to the frequency detuning parameter. As expected, at $\mathrm{A}\left(\mathrm{A}^{\prime}\right)$ and $\mathrm{C}\left(\mathrm{C}^{\prime}\right)$, the single-mode response $\left(a_{m}=0, a_{n} \neq 0\right)$ loses its stability via a subcritical pitchfork bifurcation (the solution $a_{m} \neq 0$, branching off from $a_{m}=0$, is unstable). Upon decreasing the frequency (to the left of point A) or increasing the frequency (to the right of point $\mathrm{A}^{\prime}$ ), saddle-node bifurcations occur at $\mathrm{B}$ and $\mathrm{B}^{\prime}$ where the solution $a_{m}$ becomes stable and preserves the stability over the entire range between $\mathrm{B}$ and $\mathrm{B}^{\prime}$. The only difference between the two excitation levels is that while for weak excitations (see Fig. 9 left) the high-frequency modal amplitude is always higher than the low-frequency amplitude, for harder excitations (see Fig. 9 right) there is a frequency range in which the low-frequency amplitude exceeds the high-frequency amplitude.

When the internal frequency detuning is nontrivial (see Fig. 10), the mentioned symmetry is lost although the bifurcation scenarios are preserved but for the harder excitation, which gives rise to a small frequency range in which the stable fixed point (i.e., periodic coupledmode solution) loses its stability via Hopf bifurcations at $\mathrm{E}, \mathrm{F}$ and $\mathrm{E}^{\prime}, \mathrm{F}^{\prime}$. In such a range of frequencies the response becomes quasi-periodic and may further bifurcate.
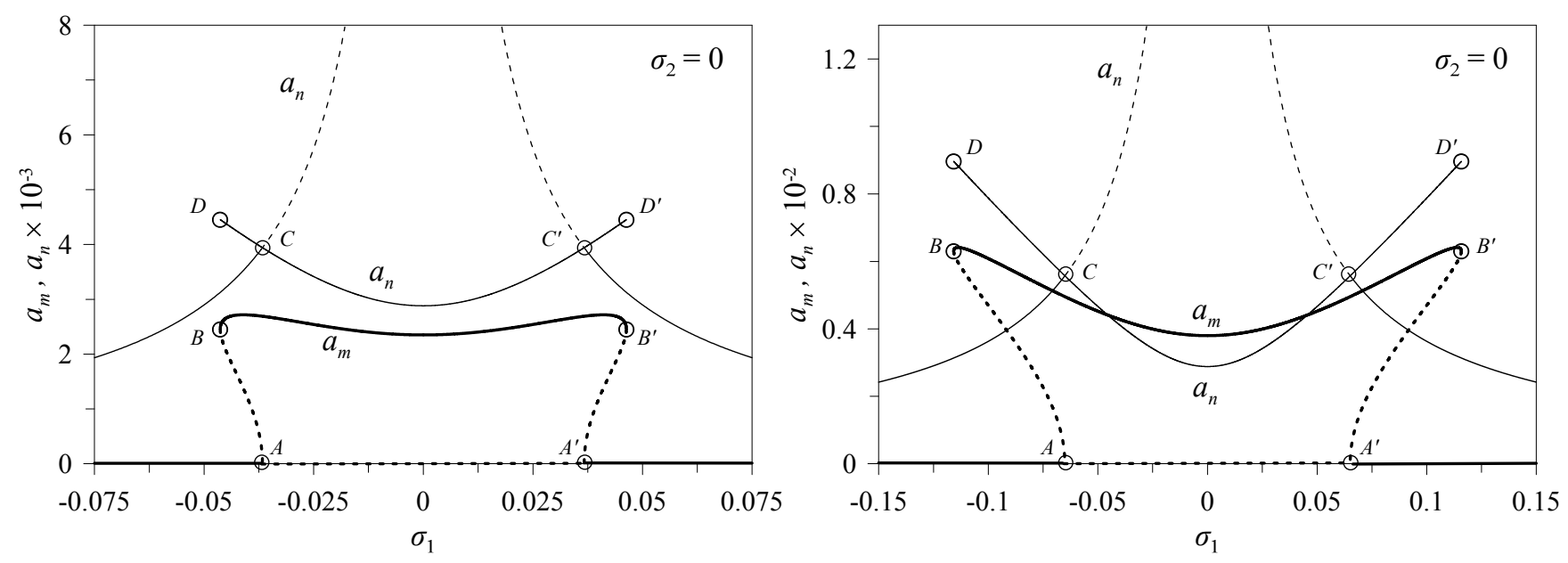

Figure 9: Second order frequency-response curves for $\sigma_{2}=0$ and (left) $\lambda=0.4 \times 10^{-3}$, (right) $\lambda=10^{-3}$.

Third order bifurcation analysis. The effects of cubic nonlinearities are studied considering 

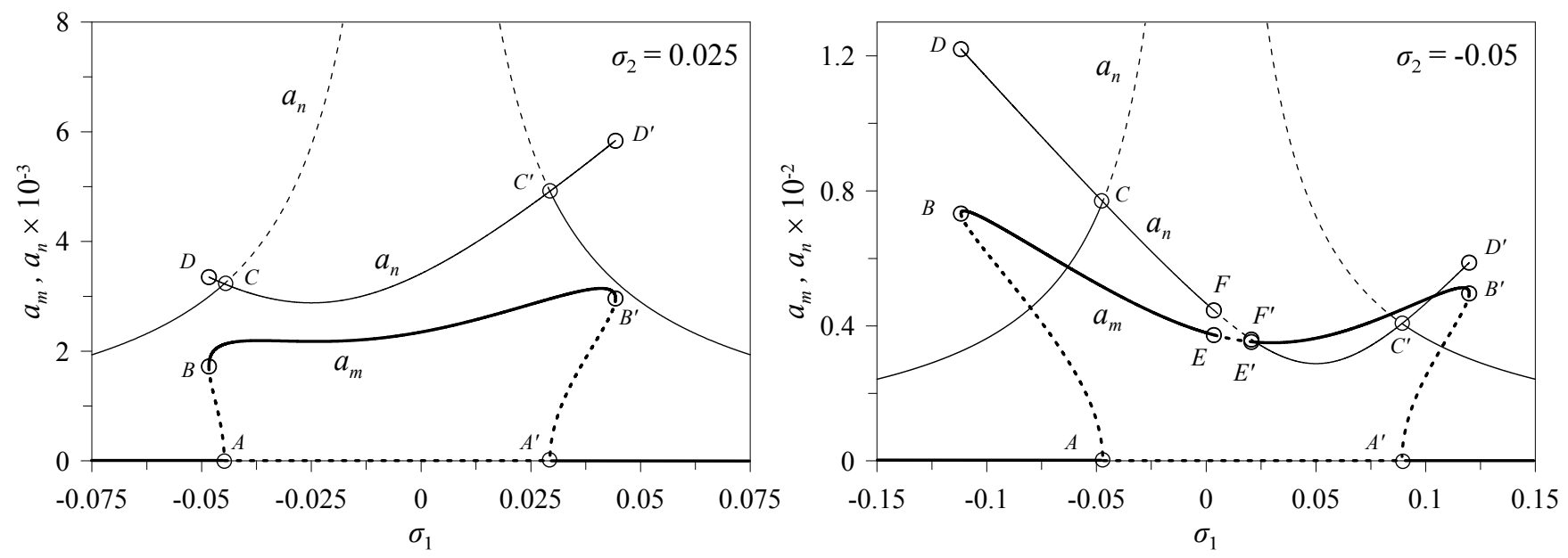

Figure 10: Second order frequency-response curves, (left) $\lambda=0.4 \times 10^{-3}$ and $\sigma_{2}=0.025$ and (right) $\lambda=10^{-3}$ and $\sigma_{2}=-0.05$.

the fixed points of the modulation equations (41) setting $\dot{a}_{m}=0, \dot{a}_{n}=0, \dot{\beta}_{1}=0$ and $\dot{\beta}_{2}=0$. To directly compare the results of the third order analysis with those of the second order case, the frequency-response curves at the same excitations levels in Fig. 9 and with perfect 2:1 tuning between the frequency of the two modes are employed. The results of the bifurcation analysis are shown in Fig. 11. Two remarkable differences are manifested: (i) the frequencyresponse curves lose the symmetry with respect to $\sigma_{1}$, (ii) there is a range of instability of the coupled-mode solution due to Hopf bifurcations between $\mathrm{E}$ and $\mathrm{E}^{\prime}, \mathrm{F}$ and $\mathrm{F}^{\prime}$. This range increases for harder excitations.

Therefore, the limitations of the low order analysis are in that (i) it misses to predict quasiperiodic solutions born out of Hopf bifurcations, (ii) it does not predict correctly the frequency ranges where the coupled-mode solution exists and its stability.

\section{Conclusions}

A mechanical model featuring the coupling between the crane deformable boom and the rigidbody dynamics of the elastically suspended container is presented. It is shown that particular configurations of the container crane system may give rise to autoparametric resonances, out of which the most powerful one seems to be the 2:1 resonance between the lowest bending mode of the boom and the lowest in-plane pendular mode of the container.

A perturbation analysis of the nonlinear interactions is carried out via the method of mul- 

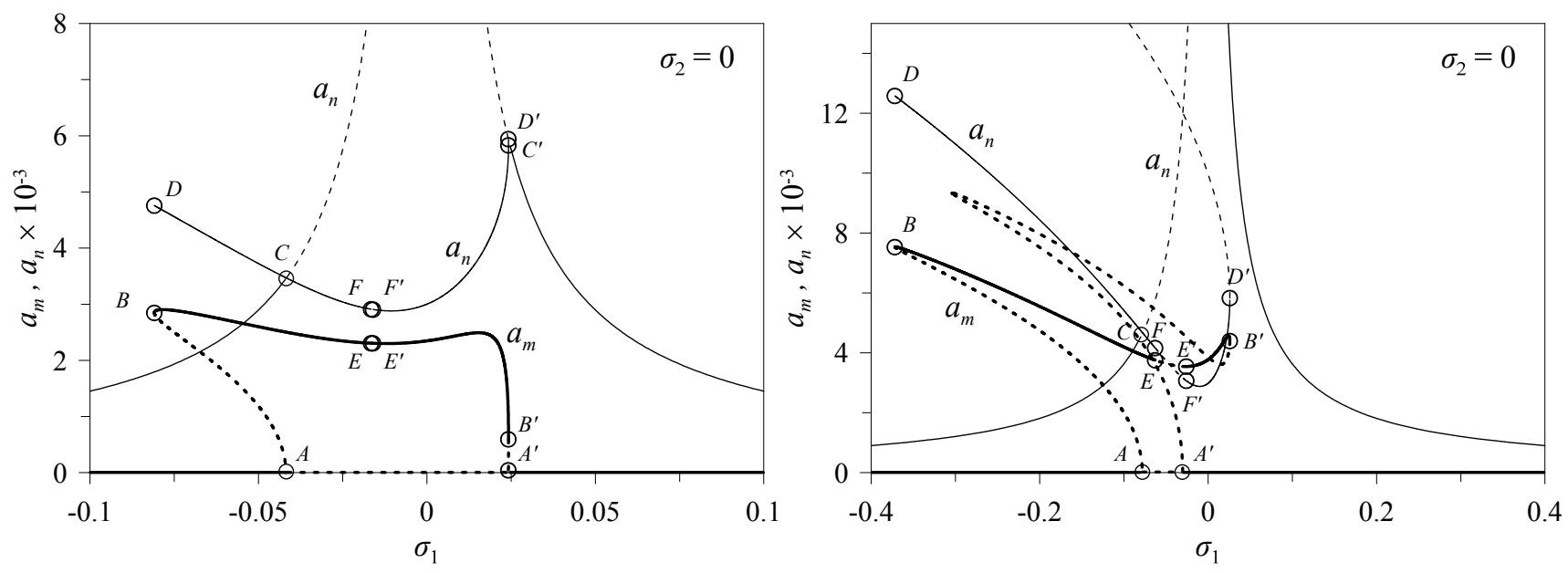

Figure 11: Third order frequency-response curves for $\sigma_{2}=0$ and (left) $\lambda=0.4 \times 10^{-3}$, (right) $\lambda=10^{-3}$.

tiple scales up to cubic order. A continuation analysis together with a stability investigation of the fixed points of the modulation equations for the amplitudes and phases of the two modes has shed light onto the bifurcation scenarios for the single-mode and coupled-mode crane responses. Differently from most of the literature on 2:1 internal resonances based on systematic truncation of the analysis to quadratic order so as to solely capture the effects of the 2:1 interaction, consideration of cubic terms shows that the frequency ranges where the coupled-mode solution exists and is stable are different from those predicted by a second order analysis. For example, considering the same excitation amplitude, the third order analysis predicts Hopf bifurcations giving rise to modulated responses of the crane (quasiperiodic flexural oscillations of the boom and quasi periodic pendular oscillations of the payload). This regime is missed by the second order analysis.

A practical implication of the obtained bifurcation results is that, given the dependence of the existence range of the coupled-mode solution from the 2:1 internal frequency detuning, the detrimental effects of the transfer of energy from the boom motion to the payload oscillations can be avoided by changing the height of the container so as to detune the frequencies of the two modes away from the 2:1 ratio. 


\section{Acknowledgements}

The financial support of Lacarbonara and Arena through the Sardinia State Research and Development Authority Grant CRP-49952 and a 2010-2011 MIUR (Italian Ministry of Education, University and Scientific Research) PRIN Grant is gratefully acknowledged.

\section{References}

[1] E. M. Abdel-Rahman, A. H. Nayfeh, and Z. Masoud. Dynamics and control of cranes: A review. Journal of Vibration and Control, 9:863-908, 2001.

[2] A. Arena, A. Casalotti, W. Lacarbonara, and M. P. Cartmell. Three-dimensional modeling of container cranes. In Proceedings of the ASME 2013 International Design Engineering Technical Conferences $\&$ Computers and Information in Engineering Conference, number DETC2013/CIE-13689, Portland, USA, August 2013.

[3] A. Arena, A. Casalotti, W. Lacarbonara, and M. P. Cartmell. Dynamics of container cranes: Three-dimensional modeling, full-scale experiments, and identification. International Journal of Mechanical Sciences, 93:8-21, 2015.

[4] C.O. Azeloglu, A. Sagirili, and A. Edincliler. Mathematical modelling of the container cranes under seismic loading and proving by shake table. Nonlinear Dynamics, 73:143$154,2013$.

[5] M. P. Cartmell. On the need for control of nonlinear oscillations in machine systems. Meccanica, 38:185212, 2003.

[6] M. P. Cartmell, L. Morrish, T. E. Alberts, and A. J. Taylor. Controlling the nonlinear dynamics of gantry cranes. Machine Vibration, 5:197210, 1996.

[7] M. P. Cartmell, L. Morrish, and A. J. Taylor. Dynamics of spreader motion in a gantry crane. IMechE Journal of Mechanical Engineering Sciences, Part C, 3(212):85-105, 1998.

[8] P. Dadone, W. Lacarbonara, A. H. Nayfeh, and H. F. VanLandingham. Payload pendulation reduction using a variable-geometry truss architecture via lqr and fuzzy controls. Journal of Vibration and Control, 9:805-837, 2003.

[9] J. B. Klaassens, G. Honderd, A. E. Azzouzi, K. C. Cheok, and G. E. Smid. 3d modeling visualization for studying controls of the jumbo container crane. In Proceedings of the American Control Conference, 1999. 
[10] W. Lacarbonara and R. Camillacci. Nonlinear normal modes of structural systems via asymptotic approach. International Journal of Solids and Structures, 41:5565-5594, 2004.

[11] W. Lacarbonara, G. Rega, and A. H. Nayfeh. Resonant non-linear normal modes. part i: analytical treatment for structural one-dimensional systems. International Journal of Non-Linear Mechanics, 38:851-872, 2003.

[12] W. Lacarbonara, R. R. Soper, A. H. Nayfeh, and D. T. Mook. A nonclassical vibration absorber for pendulation reduction. Journal of Vibration and Control, 7:365-393, 2001.

[13] Z. N. Masoud and A. H. Nayfeh. Sway reduction on container cranes using delayed feedback controller. Nonlinear Dynamics, 34:347-358, 2004.

[14] L. Meirovitch. Methods of Analytical Dynamics. McGraw-Hill, Inc., 1988.

[15] L. Morrish, M. P. Cartmell, and A. J. Taylor. Geometry and kinematics of multi-cable lifting gear. IMechE Journal of Mechanical Engineering Sciences, Part C, 3(211):185-194, 1997.

[16] A. H. Nayfeh. Nonlinear Interactions. Wiley, New York, 2000.

[17] A. H. Nayfeh and D. T. Mook. Nonlinear Oscillations. Wiley, New York, 1979.

[18] A. H. Nayfeh, R. A. Raouf, and J. F. Nayfeh. Nonlinear response of infinitely long circular cylindrical shells to subharmonic radial loads. Journal of Applied Mechanics, 58(4):10331041, 1991.

[19] X. R. Qin, Y. H. Liu, Y. Y. Liu, Q. Zhang, and Y. T. Sun. Fem and experimental modal analysis of a large-scale quayside container crane. Applied Mechanics And Materials, $387: 216-220,2013$.

[20] R. R. Soper, W. Lacarbonara, C. M. Chin, A. H. Nayfeh, and D. T. Mook. Open-loop resonance-cancellation control for a base-excited pendulum. Journal of Vibration and Control, 7:1265-1279, 2001.

[21] J.J. Wu. An Analysis of the Structural Dynamics of a Mobile Gantry Crane with Application to Automation of Container Management. PhD thesis, University of Glasgow, 2000. 